\title{
CLASS NUMBERS OF CENTRAL SIMPLE ALGEBRAS OVER GLOBAL FUNCTION FIELDS
}

\author{
FU-TSUN WEI AND CHIA-FU YU
}

\begin{abstract}
Let $K$ be a global function field together with a place $\infty$, and $A$ the subring of functions regular outside $\infty$. In this paper we present an effective method to evaluate the (locally free) class number of an arbitrary hereditary $A$-order in an arbitrary definite central simple $K$-algebra. We also show that the class number of any non-principal genus for a hereditary order in $D$ can be reduced to that of the principal genus for another hereditary order in $D$.
\end{abstract}

\section{INTRODUCTION}

Let $K$ be a global function field with finite constant field $\mathbb{F}_{q}$ together with a place $\infty$ (the place at infinity), and $A$ the subring of functions regular outside $\infty$. Let $D$ be a definite (with respect to $\infty$ ) central simple algebra over $K$ of degree $n$, that is, $D \otimes_{K} K_{\infty}$ is a division algebra with $\operatorname{dim}_{K} D=n^{2}$, where $K_{\infty}$ is the completion of $K$ at the place $\infty$. Let $R$ be a hereditary $A$-order in $D$. Recall that an $A$-order in $D$ is said to be hereditary if any left (or right) ideal of $R$ is a projective $R$-module. Denote by $h(R)$ the class number of $R$. By this we mean the number of equivalence classes of locally free right (or left) ideals of $R$. Note that for the non-commutative rings, projective modules may not be locally free. The computation of the class number $h(R)$ has been done in the following cases:

(1) The algebra $D$ is quaternion, due to Eichler [5].

(2) The algebra $D$ is of Drinfeld type, $A=\mathbb{F}_{q}[t]$ is a polynomial ring and $R$ is a maximal $A$-order, due to Gekeler [7.

(3) The algebra $D$ is of prime index and $A=\mathbb{F}_{q}[t]$ is a polynomial ring, due to Denert and Van Geel [4].

Recall that a central simple algebra $D$ over $K$ of degree $n$ is called of Drinfeld type if $D$ is ramified precisely at the place $\infty$ and another finite place $v$ with local invariants $-1 / n$ and $1 / n$, respectively. These precisely appear as endomorphism algebras of supersingular Drinfeld $A$-modules of rank $n$ over an algebraic closure $\overline{\mathbb{F}}_{v}$ of the residue field $\mathbb{F}_{v}$ of $v$.

The proof of Gekeler's class number formula is geometric. When $R$ is a maximal order, it is well-known (see [3], also cf. [6, [16]) that there is a natural bijection between the set $\mathrm{Cl}(R)$ of equivalence classes of locally free right ideals of $R$ and the set $\Lambda(n, v)$ of isomorphism classes of supersingular Drinfeld $A$-modules of rank $n$ over $\overline{\mathbb{F}}_{v}$. Using this interpretation, Gekeler calculated the number of isomorphism

Date: August 31, 2017.

2000 Mathematics Subject Classification. 11R29, 11R52,11R58, 16A18.

Key words and phrases. class number formulas, global function fields, definite central simple algebras, hereditary orders. 
classes of supersingular Drinfeld $A$-modules in the fine Drinfeld moduli spaces when $A=\mathbb{F}_{q}[t]$. Then he established the transfer principle which relates the supersingular Drinfeld $A$-modules with extra symmetries and supersingular Drinfeld $A^{\prime}$-modules of low rank for certain integral extension $A^{\prime}$ of $A$; see [7 for more details. This gives a way to express the class number $h(R)$ recursively. In [7, p. 333] Gekeler asked whether or not the transfer principle holds for a larger class of definite central simple algebras $D$ than those of Drinfeld type. We examine this in the case where the degree $n$ of $D$ is a prime number. It turns out that the (naive) transfer principle holds only for a very restricted class: only for $D$ with exactly two ramified places, $\infty$ and another finite place $v$ (but allowing different local invariants at $\infty$ and the finite ramified place $v$ ).

The proof of the class number formula for definite central division algebras of prime degree by Denert and Van Geel [4] is based on the generalization of Eichler's trace formula for Brandt matrices (see [5] and [4, Formula (7), p. 392]), and to compute explicitly the terms in the Eichler-Brandt trace formula in the case where $K$ is the rational function field. As far as the authors know, the trace formula of Brandt matrices is known only for definite central division algebras of prime degree (see [2, Theorem 4.3] and 4, Formula (7), p. 392]), and the explicit computation of the terms in the Eichler-Brandt trace formula is carried out by Denert and Van Geel only when $K$ is the rational function field (see [4, Theorems 3 and 9$]$ ).

In this article we evaluate the class number $h(R)$ for the general case, namely, for any global function field $K$ with a place at infinity, any definite central simple algebra $D$ and any hereditary $A$-order $R$ in $D$. Our approach is to construct an algebraic analogue of Gekeler's transfer principle for arbitrary definite central simple algebras, which shares the same spirit of relating class numbers of algebras of larger degrees to those of lower degrees, but allowing more complicated relations among them than the original one (as we know, the naive generalization of Gekeler's transfer principle only holds in a very restricted class). We now describe this transfer principle.

First observe that the multiplicative group $R^{\prime \times}$ of any $A$-order $R^{\prime}$ in $D$ is a finite cyclic group. More precisely, $R^{\prime \times} \simeq \mathbb{F}_{q^{s}}^{\times}$for a finite field $\mathbb{F}_{q^{s}}$ contained in $D$, and hence $s \mid n$. Let $I_{1}, \ldots, I_{h(R)}$ be representatives of locally free right ideal classes of $R$, and let $R_{i}$ be the left order of $I_{i}$ for $1 \leq i \leq h(R)$. For computing the class number $h(R)$, we may compute what we call the weight-s class number

$$
h_{s}(D / K, R)=\#\left\{i \mid 1 \leq i \leq h(R), R_{i}^{\times} \cong \mathbb{F}_{q^{s}}^{\times}\right\}
$$

for every positive divisor $s$ of $n$. A basic result says that an $A$-order $R$ is hereditary if and only if all its local completions $R_{v}$ are hereditary. Also, the hereditary order $R_{v}$ is determined by its invariant $\vec{f}_{v}=\left(f_{v, 1}, \ldots, f_{v, r_{v}}\right)$ up to conjugation; see Section 2.2. As a result, the class number $h_{s}(D / K, R)$ depends only on the invariant $\overrightarrow{\mathbf{f}}:=\left(\vec{f}_{v}\right)_{v \neq \infty}$ of $R$, so we also denote it by $h_{s}(D / K, \overrightarrow{\mathbf{f}})$.

Let $\mathbb{F}_{D}$ be a maximal finite subfield of $D$; the degree $s_{0}:=\left[\mathbb{F}_{D}: \mathbb{F}_{q}\right]$ does not depend on the choice of $\mathbb{F}_{D}$, following from the Noether-Skolem Theorem. From the basic properties, one knows

$$
h_{s}(D / K, R)=0 \quad \text { if } s \nmid s_{0} .
$$

Let $s$ be a positive divisor of $s_{0}$. Let $L_{s}:=K \cdot \mathbb{F}_{q^{s}}$ be the constant field extension of $K$ of degree $s$, and let $O_{L_{s}}$ denote the integral closure of $A$ in $L_{s}$. Choose an 
embedding $\iota: L_{s} \rightarrow D$ of $L_{s}$ into $D$, and let $D_{s}^{\prime}$ be the centralizer of its image $\iota\left(L_{s}\right)$ in $D$. We regard $L_{s}$ as a subfield of $D$ via the embedding $\iota$. Our main results (see Theorem 1.1) do not depend on the choice of the embedding $\iota$. It is easy to see that $D_{s}^{\prime}$ is again a definite central simple algebra over $L_{s}$ with respect to the unique place $\infty_{s}$ over $\infty$, with degree $n / s$ and $\left[\mathbb{F}_{D_{s}^{\prime}}: \mathbb{F}_{q^{s}}\right]=\left[\mathbb{F}_{D}: \mathbb{F}_{q}\right] / s$.

Next we define a finite index set $\Omega(D / K, s, \overrightarrow{\mathbf{f}})$. This set is actually the combinatorial description of the product of all local optimal embeddings, where we give a detailed analysis in Section 7 which is the core of our explicit computation. Let $\Sigma_{K}^{0}\left(\right.$ resp. $\left.\Sigma_{L_{s}}^{0}\right)$ the set of all finite places of $K$ (resp. of $L_{s}$ ). Let $v$ be a finite place of $K$. Let $D_{v}:=D \otimes K_{v} \simeq \operatorname{Mat}_{m_{v}}\left(\Delta_{v}\right)$, where $\Delta_{v}$ is a central division algebra over $K_{v}$ and $m_{v}$ is the local capacity at $v$ (see [11]). Let $d_{v}$ be the degree of $\Delta_{v}$; one has $n=m_{v} d_{v}$ for all $v$ and $d_{v}=1$ for almost all places $v$. Note that if $\vec{f}_{v}=\left(f_{v, 1}, \ldots, f_{v, r_{v}}\right)$ is the invariant of the local hereditary order $R_{v}$, then $\sum_{i=1}^{r_{v}} f_{v, i}=m_{v}$. Put

$$
\ell_{s, v}:=\operatorname{gcd}(s, \operatorname{deg} v), \quad \text { and } \quad t_{s, v}:=\operatorname{gcd}\left(s / \ell_{s, v}, d_{v}\right) .
$$

Let $\Omega_{v}\left(D / K, s, \vec{f}_{v}\right)$ denote the set consisting of all tuples $\left(\vec{f}_{w, *}\right)_{w \mid v}$ indexed by places $w$ of $L_{s}$ over $v$, where each $\vec{f}_{w, *}=\left(f_{w,(i, j)}\right)$ is an $r_{v} \times t_{s, v}$-matrix with nonnegative integer entries $f_{w,(i, j)} \in \mathbb{Z}_{\geq 0}\left(1 \leq i \leq r_{v}\right.$ and $\left.1 \leq j \leq t_{s, v}\right)$, that satisfy the following conditions: If one puts

$$
f_{w, i}:=\frac{s}{\ell_{s, v} t_{s, v}} \cdot \sum_{j=1}^{t_{s, v}} f_{w,(i, j)},
$$

then

$$
\sum_{i=1}^{r_{v}} f_{w, i}=\frac{m_{v}}{\ell_{s, v}} \quad \forall w \mid v, \text { and } \sum_{w \mid v} f_{w, i}=f_{v, i} \quad \text { for } 1 \leq i \leq r_{v}
$$

Then we define the index set $\Omega(D / K, s, \overrightarrow{\mathbf{f}})$ by

$$
\Omega(D / K, s, \overrightarrow{\mathbf{f}}):=\prod_{v \in \Sigma_{K}^{0}} \Omega_{v}\left(D / K, s, \vec{f}_{v}\right) .
$$

It is not hard to see that the local component $\Omega_{v}\left(D / K, s, \vec{f}_{v}\right)$ is singleton for almost all $v$ and every $\Omega_{v}\left(D / K, s, \vec{f}_{v}\right)$ is a finite set, and hence that $\Omega(D / K, s, \overrightarrow{\mathbf{f}})$ is a finite set.

We can write any element in $\Omega(D / K, s, \overrightarrow{\mathbf{f}})$ in the form $\overrightarrow{\mathbf{f}}_{*}=\left(\vec{f}_{w, *}\right)_{w \in \Sigma_{L_{s}}^{0}}$, where $\vec{f}_{w, *}=\left(f_{w,(i, j)}\right)$ is an element in $\mathbb{Z}_{\geq 0}^{r_{v}} \times \mathbb{Z}_{\geq 0}^{t_{s, v}}$ with the conditions above. We make an appropriate order on the index set $\{(i, j)\}$ and regard $\vec{f}_{w, *}$ as a (long) vector in $\mathbb{Z}_{\geq 0}^{r_{v} \cdot t_{s, v}}$; see Section 4.2 for details. Denote by $\vec{f}_{w, *}^{o}$ the vector obtained by removing zero entries of the vector $\vec{f}_{w, *}$ (also see Section 4.2) and define $\overrightarrow{\mathbf{f}}_{*}^{o}:=\left(\vec{f}_{w, *}^{o}\right)_{w \in \Sigma_{L_{s}}^{o}}$. Note that the sum $\sum_{i, j} f_{w, i, j)}$ is equal to the local capacity of the central simple algebra $D_{s}^{\prime}$ over $L_{s}$ at $w$ for all finite places $w$. Therefore, there is a hereditary order $R^{\prime}\left(\overrightarrow{\mathbf{f}}_{*}^{o}\right)$ in $D_{s}^{\prime}$ with invariant $\overrightarrow{\mathbf{f}}_{*}^{o}$ and it makes sense to talk about the class numbers $h_{s^{\prime \prime}}\left(D_{s}^{\prime} / L_{s}, \overrightarrow{\mathbf{f}}_{*}^{o}\right):=h_{s^{\prime \prime}}\left(D_{s}^{\prime} / L_{s}, R^{\prime}\left(\overrightarrow{\mathbf{f}}_{*}^{o}\right)\right)$ for positive divisors $s^{\prime \prime}$ of $\left[\mathbb{F}_{D_{s}^{\prime}}: \mathbb{F}_{q^{s}}\right]$.

With notations being as above, our transfer principle is stated as follows. 
Theorem 1.1. For any two positive divisors $s$ and $s^{\prime}$ of $\left[\mathbb{F}_{D}: \mathbb{F}_{q}\right]$ with $s \mid s^{\prime}$, we have

$$
s \cdot h_{s^{\prime}}(D / K, R)=\sum_{\overrightarrow{\mathbf{f}}_{*} \in \Omega(D / K, s, \overrightarrow{\mathbf{f}})} h_{s^{\prime} / s}\left(D_{s}^{\prime} / L_{s}, R^{\prime}\left(\overrightarrow{\mathbf{f}}_{*}^{o}\right)\right) .
$$

We make a few remarks about Theorem 1.1. When $D$ is of Drinfeld type, Theorem 1.1 recovers Gekeler's transfer principle (cf. Theorem 5.1). See Section 5.1 for the explanation of Gekeler's transfer principle for supersingular Drinfeld modules and the deduction from Theorem 1.1. As a result, we give an algebraic proof of Theorem 5.1. Note that in this special case, the hereditary orders $R^{\prime}\left(\overrightarrow{\mathbf{f}}_{*}^{o}\right)$ in $D_{s}^{\prime}$ occurred in (1.4) are also maximal. This is not true for the general cases; one needs to deal with class numbers of hereditary orders (in smaller subalgebras) as well even when one starts with a maximal order $R$ in $D$. This is also a main reason for us to consider directly the cases of arbitrary hereditary orders.

Theorem 1.1 indicates that in order to get $h_{s}(D / K, R)$, it suffices to compute $h_{1}\left(D_{s}^{\prime} / L_{s}, R^{\prime}\left(\overrightarrow{\mathbf{f}}_{*}^{o}\right)\right)$ for each $\overrightarrow{\mathbf{f}}_{*}$ in $\Omega(D / K, s, \overrightarrow{\mathbf{f}})$. Recall that the associated mass $\operatorname{Mass}(D / K, R)$ is defined as follows:

$$
\operatorname{Mass}(D / K, R):=\sum_{1 \leq i \leq h(R)} \frac{1}{\#\left(R_{i}^{\times}\right)}=\sum_{s \mid\left[\mathbb{F}_{D}: \mathbb{F}_{q}\right]} \frac{h_{s}(D / K, R)}{q^{s}-1},
$$

where $R_{i}$ is the left order of each $R$-ideal $I_{i}$. The mass $\operatorname{Mass}(D / K, R)$ only depends on the invariant $\overrightarrow{\mathbf{f}}$ of $R$, so we may also denote it by $\operatorname{Mass}(D / K, \overrightarrow{\mathbf{f}})$. Notice that $\#\left(R_{i}^{\times}\right)=q^{s}-1$ if there exists an optimal embedding of $O_{L_{s}}$ into $R_{i}$. The mass formula (cf. [4, the precise formula is also stated in Theorem 3.1) shows that the mass $\operatorname{Mass}(D / K, R)$ can be computed explicitly in terms of integral values of the zeta function of $K$. Theorem 1.1 and the mass formula for $\operatorname{Mass}\left(D_{s}^{\prime} / L_{s}, R^{\prime}\left(\overrightarrow{\mathbf{f}}_{*}^{o}\right)\right)$ together provide enough equations to solve each weight- $s$ class number $h_{s}(D / K, R)$. As a result, the class number $h(R)$ can be also expressed in terms of special zeta values eventually.

The second part of main results is to make the computation of the class number more effectively. Note that the index set $\Omega(D / K, s, \overrightarrow{\mathbf{f}})$ in (1.4) is the product of its local components $\Omega_{v}(1.3)$. However, the class number $h_{s^{\prime} / s}\left(D_{s}^{\prime} / L_{s}, R^{\prime}\left(\overrightarrow{\mathbf{f}}_{*}^{o}\right)\right)$ is not. Therefore, there is no direct way to reduce the computations locally. The way we do is to regroup the relation (1.4) into the (partial) mass sums (see Theorem 4.6):

$$
s \cdot \sum_{s^{\prime}: s\left|s^{\prime}\right|\left[\mathbb{F}_{D}: \mathbb{F}_{K}\right]} \frac{h_{s^{\prime}}(D / K, \overrightarrow{\mathbf{f}})}{q^{s^{\prime}}-1}=\sum_{\overrightarrow{\mathbf{f}}_{*} \in \Omega(D / K, s, \overrightarrow{\mathbf{f}})} \operatorname{Mass}\left(D_{s}^{\prime} / L_{s}, \overrightarrow{\mathbf{f}}_{*}^{o}\right) .
$$

Note that now one can compute the right side of the equation (1.5) directly using the mass formula, without going through the induction step of computing $h_{1}\left(D_{s}^{\prime} / L_{s}, R^{\prime}\left(\overrightarrow{\mathbf{f}}_{*}^{o}\right)\right)$ recursively. This simplifies the computation significantly.

The second input is using the nice property of the masses, which allows us to separate the global and local contributions. Set

$$
\operatorname{Mass}\left(D_{s}^{\prime} / L_{s}\right):=\operatorname{Mass}\left(D_{s}^{\prime} / L_{s}, \overrightarrow{\mathbf{f}}_{\max }^{\prime}\right)
$$


where $\overrightarrow{\mathbf{f}}_{\max }^{\prime}$ is the invariant of a maximal $O_{L_{s}}$-order $R_{\max }^{\prime}$ in $D_{s}^{\prime}$. For any finite place $w$ of $L_{s}$ and any vector $\vec{h}_{w}=\left(h_{w, 1}, \ldots, h_{w, r_{w}}\right) \in \mathbb{Z}_{\geq 0}^{r_{w}}$, we set

$$
\mathcal{T}^{\prime}{ }_{w}\left(D_{s}^{\prime} / L_{s}, \vec{h}_{w}\right):=\frac{\prod_{i=1}^{m_{w}}\left(N(w)^{d_{w} i}-1\right)}{\prod_{i=1}^{r_{w}}\left(\prod_{j=1}^{h_{v, i}}\left(N(w)^{d_{w} j}-1\right)\right)},
$$

where $m_{w}$ is the local capacity of $D_{s}^{\prime}$ at $w, d_{w}$ is the local index of $D_{s}^{\prime}$ at $w$ and $N(w)$ is the cardinality of the residue field of $L_{s}$ at $w$. Then the mass formula (Theorem 3.1) gives

$$
\left.\operatorname{Mass}\left(D_{s}^{\prime} / L_{s}, \overrightarrow{\mathbf{f}}_{*}^{o}\right)\right)=\operatorname{Mass}\left(D_{s}^{\prime} / L_{s}\right) \cdot \prod_{w \in \Sigma_{L_{s}}^{0}} \mathcal{T}^{\prime}{ }_{w}\left(D_{s}^{\prime} / L_{s}, \vec{f}_{w, *}\right) .
$$

For each finite place $v$ of $K$, define

$$
\Theta_{v}\left(D / K, s, \vec{f}_{v}\right):=\sum_{\left(\vec{f}_{w, *}\right)_{w \mid v} \in \Omega_{v}\left(D / K, s, \vec{f}_{v}\right)}\left(\prod_{w \mid v} \mathcal{T}^{\prime}{ }_{w}\left(D_{s}^{\prime} / L_{s}, \vec{f}_{w, *}\right)\right) .
$$

Our second main result (Theorem 4.7) states as follows, which reduces the computation in purely local terms.

Theorem 1.2. Notations being as above, one has

$$
s \cdot \sum_{s^{\prime}: s\left|s^{\prime}\right|\left[\mathbb{F}_{D}: \mathbb{F}_{K}\right]} \frac{h_{s^{\prime}}(D / K, \overrightarrow{\mathbf{f}})}{q^{s^{\prime}}-1}=\operatorname{Mass}\left(D_{s}^{\prime} / L_{s}\right) \cdot \prod_{v \in \Sigma_{K}^{0}} \Theta_{v}\left(D / K, s, \vec{f}_{v}\right) .
$$

We also provide a simple method to compute the local terms $\Theta_{v}\left(D / K, s, \vec{f}_{v}\right)$ using generating functions (see Proposition 4.8). In Section 4.5 we present a recursive formula for computing the class number $h(R)$ using Theorems 1.1 and 1.2 together with the explicit computation of the local terms $\Theta_{v}\left(D / K, s, \vec{f}_{v}\right)$.

We already mentioned that the index set $\Omega(D / K, s, \overrightarrow{\mathbf{f}})$ is the combinatorial description of the product of all local optimal embeddings of $L_{s}$ in $R_{i}$, following from the results in Section 7 Therefore, this gives a combinatorial criterion of determining the existence of optimal embeddings. As a by-product, we obtain the following generalization (Theorem 4.4) of Eichler's theorem [5] on optimal embeddings from quaternion algebras to central simple algebras, which is of interest in its own right.

Theorem 1.3. Notations being as above. There is an optimal embedding of $O_{L_{s}}$ into a hereditary order $R^{\prime}$ in $D$ of invariant $\overrightarrow{\mathbf{f}}$ if and only if for all $v \in \Sigma_{K}^{0}$, one has the divisibility

$$
\frac{s}{\ell_{s, v} t_{s, v}} \mid f_{v, i}
$$

for $1 \leq i \leq r_{v}$.

In 8] Papikian establishes a bijection between the isomorphism classes of exceptional $\mathcal{D}$-elliptic sheaves with the ideal classes of a certain hereditary order. Also, he identified the isomorphism classes of supersingular $\mathcal{D}$-elliptic sheaves with the ideal classes of a maximal order. One application of our main results evaluates the number of isomorphism classes of those $\mathcal{D}$-elliptic sheaves. 
Another application of our results is to compute the dimension of certain automorphic forms for definite central simple algebras. Indeed, the class number $h(R)$ is simply the dimension of the space $L^{2}\left(D^{\times} \backslash \widehat{D}^{\times} / \widehat{R}^{\times}\right)$of automorphic forms. Here $\widehat{D}:=D \otimes_{K} \mathbb{A}_{K}^{\infty}$ and $\widehat{R}:=R \otimes_{A} \widehat{A}$, where $\mathbb{A}_{K}^{\infty}$ is the ring of finite adeles of $K$ and $\widehat{A}$ is the pro-finite completion of $A$.

This paper is organized as follows. In Section 2, we include the preliminaries for central simple algebras over global function fields and local properties of hereditary orders. We also compute the degree $\left[\mathbb{F}_{D}: \mathbb{F}_{q}\right]$ of a maximal finite subfield $\mathbb{F}_{D}$ in $D$. In Section 3 we explain the strategy of computing the class number from the mass formula and optimal embeddings. An explicit mass formula for hereditary $A$-orders is described in Section 3.1. In Section 3.2 we translate the problem of computing class numbers into that for numbers of (global) optimal embeddings. We also note at the end of Section 3 that global optimal embeddings can be understood through the study of local optimal embeddings. The main theorems are presented in Section 4, and we give a recursive formula for computing the class number in Section 4.5. Section 5 includes special cases and examples to show the relation between the main theorems and previous results in [4 and [6]. In Section [5.1] we recall Gekeler's transfer principle for supersingular Drinfeld modules and deduce Gekeler's result from Theorem [1.1. In Section [5.2, we focus on the special case where $\left[\mathbb{F}_{D}: \mathbb{F}_{q}\right]$ is a prime, and express the class number $h(R)$ in terms of masses. Moreover, when the degree $n$ of $D$ over $K$ is a prime number, we obtain an explicit class number formula which coincides with the formulas in [4, Theorems 3 and 9] in the case where $K$ is the rational function field. We also give an example for the reader's interest using the recursive formula of Section 4.5. In Section 6 we show that the computation of the class number of other genus of (not necessarily locally free) $R$-ideals can be reduced to that of locally free $R^{\prime}$-ideals for another hereditary $A$-order $R^{\prime}$, the principal genus class number. Section 7 is an independent section, in which we make a detailed study of local optimal embeddings. This also plays the key ingredient in the proof of our main theorem Theorem 1.1

\section{Preliminaries}

In this section, we set up general notations and establish basic results of definite central simple algebras of arbitrary degree which we shall need in this article. Further details are referred to [1].

2.1. General settings. Let $K$ be a global function field with finite constant field $\mathbb{F}_{q}$, i.e. the transcendental degree of $K$ over $\mathbb{F}_{q}$ is one and $\mathbb{F}_{q}$ is algebraically closed in $K$. For each place $v$ of $K$, the completion of $K$ at $v$ is denoted by $K_{v}$, and we set $O_{v}$ to be the valuation ring of $K_{v}$. Fix a uniformizer $\pi_{v}$ in $O_{v}$. The residue field $O_{v} /\left(\pi_{v}\right)$ is denoted by $\mathbb{F}_{v}$, and $\operatorname{deg} v$ is the degree of $\mathbb{F}_{v}$ over $\mathbb{F}_{q}$. There is a canonical embedding $\mathbb{F}_{v} \hookrightarrow O_{v}$, and $O_{v}$ is in fact isomorphic to the power series ring $\mathbb{F}_{v}\left[\left[\pi_{v}\right]\right]$. The cardinality of $\mathbb{F}_{v}$ is denoted by $N(v)$.

Let $D$ be a central simple algebra over $K$ with $\operatorname{dim}_{K} D=n^{2}$. For each place $v$ of $K$, we have that $D_{v}:=D \otimes_{K} K_{v}$ is isomorphic to $\operatorname{Mat}_{m_{v}}\left(\Delta_{v}\right)$, where $\Delta_{v}$ is a central division algebra over $K_{v}$ with $\operatorname{dim}_{K_{v}} \Delta_{v}=d_{v}^{2}$ and $m_{v} d_{v}=n$. We recall the definition of local invariants of $D$. Note that $\Delta_{v}$ contains an unramified maximal 
subfield $F_{v}$ (so $\left[F_{v}: K_{v}\right]=d_{v}$ ), and there exists an element $u_{v}$ in $\Delta_{v}$ such that $u_{v}^{d_{v}}=\pi_{v}^{\kappa_{v}}$ where $\kappa_{v} \in \mathbb{Z}$ with $\operatorname{gcd}\left(\kappa_{v}, d_{v}\right)=1$, and

$$
u_{v} \alpha_{v}=\operatorname{Fr}_{v}\left(\alpha_{v}\right) u_{v} \text { for all } \alpha_{v} \text { in } F_{v} .
$$

Here $\operatorname{Fr}_{v}$ is the Frobenius automorphism of $F_{v}$ over $K_{v}$, i.e. for any $\alpha_{v}$ in the valuation ring $O_{F_{v}}$ of $F_{v}$ we have $\operatorname{Fr}_{v}\left(\alpha_{v}\right) \equiv \alpha_{v}^{N(v)}\left(\bmod \pi_{v} O_{F_{v}}\right)$. The local invariant $\operatorname{inv}_{v}(D)$ of $D$ at $v$ is defined as

$$
\operatorname{inv}_{v}(D):=\kappa_{v} / d_{v} \bmod \mathbb{Z} \in \mathbb{Q} / \mathbb{Z}
$$

which is independent of the choices of $F_{v}, \pi_{v}$, and $u_{v}$. We call $D$ ramified at $v$ if $\operatorname{inv}_{v}(D) \not \equiv 0(\bmod \mathbb{Z})$, i.e. $\Delta_{v}$ is not equal to $K_{v}$. Let $S=S_{D}$ be the set consisting of places of $K$ where $D$ is ramified. It is well-known that $S$ is finite, and that

$$
\sum_{v \in V^{K}} \operatorname{inv}_{v}(D) \equiv 0 \quad(\bmod \mathbb{Z}),
$$

where $\Sigma_{K}$ denotes the set of all places of $K$.

2.2. Hereditary orders. Fix a place $\infty$ of $K$, referred as the place at infinity; and others are referred as finite places of $K$. Let $A$ be the ring of functions in $K$ regular outside $\infty$. Recall that a hereditary $A$-order $R$ in $D$ is an $A$-order in $D$ such that every left (or equivalently right) ideal of $R$ is projective as an $R$-module. It is known that an $A$-order $R$ in $D$ is hereditary if and only if its completion $R_{v}:=R \otimes_{A} O_{v}$ is hereditary for all finite places $v$ of $K$.

Let $R$ be a hereditary $A$-order in $D$. For each finite place $v$ of $K$. The completion $R_{v}:=R \otimes_{A} O_{v}$ is a hereditary $O_{v}$-order in $D_{v} \cong \operatorname{Mat}_{m_{v}}\left(\Delta_{v}\right)$. The unique maximal $O_{v}$-order in $\Delta_{v}$ is denoted by $O_{\Delta_{v}}$ and we set $\mathfrak{P}_{v}$ to be its maximal (two-sided) ideal. It is known that there exists a vector $\vec{f}_{v}=\left(f_{v, 1}, \ldots, f_{v, r_{v}}\right)$, where $f_{v, 1}, \ldots, f_{v, r_{v}}$ are positive integers such that $\sum_{i=1}^{r_{v}} f_{v, i}=m_{v}$, and $R_{v}$ is isomorphic to the ring $\operatorname{Mat}_{m_{v}}\left(\vec{f}_{v}, O_{\Delta_{v}}\right)$ consisting of elements $X=\left(X_{i, j}\right)_{1 \leq i, j \leq r_{v}}$ in $\operatorname{Mat}_{m_{v}}\left(O_{\Delta_{v}}\right)$ such that

$$
X_{i, j} \in \begin{cases}\operatorname{Mat}_{f_{v, i} \times f_{v, j}}\left(O_{\Delta_{v}}\right) & \text { if } i \leq j, \\ \operatorname{Mat}_{f_{v, i} \times f_{v, j}}\left(\mathfrak{P}_{v}\right) & \text { if } i>j .\end{cases}
$$

The number $r_{v}$ is called the period of $R_{v}$; the vector $\vec{f}_{v}:=\left(f_{v, 1}, \ldots, f_{v, r_{v}}\right)$ is called the invariant of $R$ at $v$, which is uniquely determined by $R_{v}$ up to cyclic permutations. When $\mathbf{f}_{v}=(1, \ldots, 1)$, the order $R_{v}$ is the Iwahori order which is the preimage of the set of upper triangular matrices over $O_{\Delta_{v}} / \mathfrak{P}_{v}$. We shall also call the collection $\mathbf{f}=\left(\mathbf{f}_{v}\right)_{v \neq \infty}$ the invariant of $R$.

Note that the class number $h(R)$ only depends on the invariant $\mathbf{f}$ of $R$ but not on $R$ itself. We also write $h(D / K, \mathbf{f})$ for the class number number $h(R)$.

2.3. The constant field of $D$. Let $K, \infty, A$ and $D$ be as above. Assume that $D$ is definite with respect to $\infty$, that is, the completion $D_{\infty}:=D \otimes_{K} K_{\infty}$ at $\infty$ is a division algebra. For any element $\alpha$ in $D$ which is algebraic over $\mathbb{F}_{q}, \mathbb{F}_{q}(\alpha)$ is a finite field with

$$
\left[\mathbb{F}_{q}(\alpha): \mathbb{F}_{q}\right]=[K(\alpha): K] \mid n,
$$

as $K(\alpha)$ is a subfield of $D$.

Let $s$ be a positive divisor of $n$. Put $L_{s}:=K \mathbb{F}_{q^{s}}$, the constant field extension of $K$ of degree $s$. For any place $v$ of $K$, let $\ell_{s, v}$ be the number of places of $L_{s}$ over 
$v$. One has $\ell_{s, v}=\operatorname{gcd}(s, \operatorname{deg} v)$. The following lemma gives the criterion for the existence of an embedding of $L_{s}$ into $D$.

Lemma 2.1. There exists an embedding $\iota: L_{s} \hookrightarrow D$ if and only if $\ell_{s, v}$ divides $m_{v}$ for all places $v$ of $K$.

Proof. Suppose $D \otimes_{K} L_{s} \cong \operatorname{Mat}_{c_{s}}\left(\Delta_{s}\right)$, where $\Delta_{s}$ is a central division algebra over $L_{s}$. By [12, Lemma 2.3], there exists an embedding of $L_{s}$ into $D$ if and only if $s \mid c_{s}$. For each place $v$ of $K$, let $w$ be a place of $L_{s}$ lying above $v$. We denote by $L_{s, w}$ the completion of $L_{s}$ at $w$; one has $\left[L_{s, w}: K_{v}\right]=s / \ell_{s, v}$. Write

$$
D \otimes_{K} L_{s, w} \cong \operatorname{Mat}_{r_{w}}\left(\Delta_{w}^{\prime}\right),
$$

where $\Delta_{w}^{\prime}$ is a central division algebra over $L_{s, w}$. Then by [11, (31.9) Theorem] (remembering $D_{v} \simeq \operatorname{Mat}_{m_{v}}\left(\Delta_{v}\right)$ ),

$$
r_{w}=m_{v} \cdot \operatorname{gcd}\left(d_{v},\left[L_{s, w}: K_{v}\right]\right)=\operatorname{gcd}\left(m_{v} d_{v}, m_{v} \cdot \frac{s}{\ell_{s, v}}\right)=\operatorname{gcd}\left(n, s \cdot \frac{m_{v}}{\ell_{s, v}}\right) .
$$

It is known that (cf. [11, (32.17) Theorem])

$$
c_{s}=\operatorname{gcd}\left(r_{w}: \text { place } w \text { of } L_{s}\right) .
$$

It follows from (2.1) that $s \mid r_{w}$ if and only if $\ell_{s, v} \mid m_{v}$, and (2.2) says that $s \mid c_{s}$ if and only if $s \mid r_{w}$. This completes the proof of the lemma.

Definition 2.2. Let $s_{0}$ be the divisor of $n$ which is maximal such that $\ell_{s_{0}, v}$ divides $m_{v}$ for all places $v$ of $K$. Then the finite field $\mathbb{F}_{D}:=\mathbb{F}_{q^{s_{0}}}$ is called the constant field of $D$.

Remark. (1) By Lemma 2.1, any maximal finite subfield of $D$ is isomorphic to $\mathbb{F}_{D}$. (2) For our convenience, we also denote by $\mathbb{F}_{K}$ the constant field of $K$.

Suppose $n=p_{1}^{n_{1}} \ldots p_{r}^{n_{r}}$, where $p_{i}$ are distinct prime numbers and $n_{i}$ are positive integers. For any $v \in \Sigma_{K}$, the set of all places of $K$, let

$$
s=\prod_{i=1}^{r} p_{i}^{n_{i}(s)}, \quad m_{v}=\prod_{i=1}^{r} p_{i}^{m_{i}(v)}, \quad \text { and } \quad \operatorname{gcd}(\operatorname{deg} v, n)=\prod_{i=1}^{r} p_{i}^{n_{i}(v)}
$$

be the primary decomposition. Then one has $\ell_{s, v} \mid m_{v}$ if and only if

$$
\min \left\{n_{i}(s), n_{i}(v)\right\} \leq m_{i}(v), \quad \forall i=1, \ldots, r .
$$

Note that $m_{i}(v)=n_{i}$ for almost all places $v \in \Sigma_{K}$. For each $i=1, \ldots, r$, if $n_{i}(v) \leq m_{i}(v)$ for all $v \in \Sigma_{K}$, then $n_{i}(s)$ can be any integer $0 \leq n_{i}(s) \leq n_{i}$. Let $S_{i}:=\left\{v \in S \mid n_{i}(v)>m_{i}(v)\right\}$. Then if $S_{i}$ is non-empty, one has

$$
0 \leq n_{i}(s) \leq \min _{v \in S_{i}}\left\{m_{i}(v)\right\} .
$$

Therefore, we obtain the following lemma which computes the degree $s_{0}=\left[\mathbb{F}_{D}\right.$ : $\left.\mathbb{F}_{K}\right]$.

Lemma 2.3. Let the notation be as above and let $s_{0}=\prod_{i=1}^{r} p_{i}^{n_{i}\left(s_{0}\right)}$. Then one has for $i=1, \ldots, r$,

$$
n_{i}\left(s_{0}\right)= \begin{cases}\min _{v \in S_{i}}\left\{m_{i}(v)\right\}, & \text { if } S_{i} \neq \emptyset, \\ n_{i}, & \text { otherwise }\end{cases}
$$


Now, let $R$ be an $A$-order in $D$. Then the multiplicative group $R^{\times}$must be a finite cyclic group. More precisely:

Lemma 2.4. Let the notation and assumption be as above and let $R$ be any $A$ order in D. Then the multiplicative group $R^{\times}$is isomorphic to $\mathbb{F}_{q^{s}}^{\times}$for some positive integer $s \mid\left[\mathbb{F}_{D}: \mathbb{F}_{K}\right]$.

Proof. Since $R$ is discrete in $D_{\infty}$ and $R^{\times}$is in the maximal compact subring $O_{D_{\infty}}$ of $D_{\infty}$, we have the finiteness of $R^{\times}$. We show that the group homomorphism

$$
R^{\times} \longrightarrow\left(O_{D_{\infty}} / \mathfrak{P}_{\infty}\right)^{\times} \text {. }
$$

is injective. Let $a \in R^{\times}$be an element in the kernel. Then $a=1+\alpha$ where $\alpha \in \mathfrak{P}_{\infty}$. The finiteness of the order of $a$ implies that $\alpha=0$. To see this, suppose the order of $a=p^{r} \cdot m$ where $p$ is the characteristic of $\mathbb{F}_{q}$ and $\operatorname{gcd}(p, m)=1$. Write $a^{p^{r}}=1+b \Pi$ where $b \in O_{\Delta_{\infty}}$ and $\Pi$ is a generator of $\mathfrak{P}_{\infty}$. Then

$$
1=\left(a^{p^{r}}\right)^{m}=1+m \cdot b \Pi+\cdots
$$

and hence $b=0$. Thus we have $a^{p^{r}}=1$, i.e. $(\alpha)^{p^{r}}=0$. Since $D$ is a division algebra, $\alpha=0$.

Therefore $R^{\times}$is a finite cyclic group, and $\mathbb{F}_{q}\left[R^{\times}\right] \simeq \mathbb{F}_{q^{s}}$ for some positive integer $s \mid s_{0}=\left[\mathbb{F}_{D}: \mathbb{F}_{q}\right]$. Since $\mathbb{F}_{q}\left[R^{\times}\right] \subset R$, one has

$$
R^{\times}=\left(\mathbb{F}_{q}\left[R^{\times}\right]\right)^{\times} \simeq \mathbb{F}_{q^{s}}^{\times} .
$$

This completes the proof of the lemma.

\section{Strategy of COmputing the Class Number $h(R)$}

In this section, we explain how to compute the class number $h(R)$ of a hereditary $A$-order $R$ in $D$. Recall that $D$ is a definite central simple algebra over $K$ with $\operatorname{dim}_{K} D=n^{2}$ and $R$ is a hereditary $A$-order in $D$.

3.1. Mass formulas. A locally free (fractional) right ideal $I$ of $R$ is a projective $A$-lattice in $D$ such that $I \cdot R=I$ and for each finite place $v$ of $K$, there exists $\alpha_{v}$ in $D_{v}^{\times}$such that $I_{v}\left(:=I \otimes_{A} O_{v}\right)=\alpha_{v} R_{v}$. Two locally free right ideals $I_{1}$ and $I_{2}$ are called equivalent if there exists an element $b$ in $D^{\times}$such that $I_{1}=b \cdot I_{2}$. We are interested in the number $h=h(R)$ of locally free right ideal classes of $R$ in $D$.

Let $\mathbb{A}_{K}^{\infty}$ be the ring of finite adeles of $K$, and let $\widehat{A}$ be the pro-finite completion of $A$. Note that the set of locally free right ideal classes of $R$ can be identified with the finite double coset space $D^{\times} \backslash \widehat{D}^{\times} / \widehat{R}^{\times}$, where $\widehat{D}:=D \otimes_{K} \mathbb{A}_{K}^{\infty}$ and $\widehat{R}:=R \otimes_{A} \widehat{A}$. More precisely, let $g_{1}, \ldots, g_{h}$ be representatives of the double cosets. Then $\left\{I_{i}:=\right.$ $\left.D \cap g_{i} \widehat{R} \mid 1 \leq i \leq h\right\}$ is a set of representatives of locally free right ideal classes of $R$.

For $1 \leq i \leq h$, let $R_{i}$ be the left order of $I_{i}$. Since $D$ is definite, by Lemma 2.4 the cardinality of the multiplicative group $R_{i}^{\times}$is finite. The mass sum $\operatorname{Mass}(D / K, R)$ is defined by

$$
\operatorname{Mass}(D / K, R):=\sum_{1 \leq i \leq h} \frac{1}{\#\left(R_{i}^{\times}\right)} .
$$

Let $S^{\prime}=S_{R}^{\prime}$ be the set of finite places $v$ of $K$ for which $R_{v}$ is not a maximal $O_{v}$-order in $D_{v}$. Recall that $S=S_{D}$ is the set of ramified places (including $\infty$ ) of 
$K$ for $D$. The mass sum Mass $(D / K, R)$ has an explicit description by the following theorem:

Theorem 3.1. (Mass formula) Let $D$ be a definite central simple algebra over $K$ with $\operatorname{dim}_{K} D=n^{2}$. Let $R$ be a hereditary A-order in $D$. For each place $v$ of $K$. Suppose the local invariant $\operatorname{inv}_{v}(D)$ is $\kappa_{v} / d_{v} \bmod \mathbb{Z}$ and the invariant of $R$ at $v$ (when $v$ is a finite place of $K$ ) is $\vec{f}_{v}=\left(f_{v, 1}, \ldots, f_{v, r_{v}}\right)$. Then we have

$$
\operatorname{Mass}(D / K, R)=\frac{\# \operatorname{Pic}(A)}{q-1} \cdot \prod_{i=1}^{n-1} \zeta_{K}(-i) \cdot \prod_{v \in S} \mathcal{T}_{v} \cdot \prod_{v \in S^{\prime}} \mathcal{T}_{v}^{\prime},
$$

where $\operatorname{Pic}(A)$ is the Picard group of $A, \zeta_{K}(s)$ is the Dedekind zeta function of $K$ :

$$
\zeta_{K}(s):=\prod_{v \in \Sigma_{K}}\left(1-N(v)^{-s}\right)^{-1},
$$

the constants $\mathcal{T}_{v}$ and $\mathcal{T}_{v}^{\prime}$ are given by

$$
\mathcal{T}_{v}=\prod_{\substack{1 \leq i \leq n-1, d_{v} \dagger i}}\left(N(v)^{i}-1\right)
$$

and

$$
\mathcal{T}_{v}^{\prime}:=\left[\mathrm{GL}_{m_{v}}\left(O_{\Delta_{v}}\right): R_{v}^{\times}\right]=\frac{\prod_{i=1}^{m_{v}}\left(N(v)^{d_{v} i}-1\right)}{\prod_{i=1}^{r_{v}}\left(\prod_{j=1}^{f_{v, i}}\left(N(v)^{d_{v} j}-1\right)\right)} .
$$

Proof. See [4, p. 382] and 9]. Also see [14 for detailed computations of the proof.

3.2. Optimal embeddings. Let $\mathbb{F}_{D}$ be the constant field of $D$. We have shown that $R_{i}^{\times} \cong \mathbb{F}_{q^{s_{i}}}^{\times}$for some $s_{i} \mid s_{0}$ where $s_{0}=\left[\mathbb{F}_{D}: \mathbb{F}_{K}\right]$. Set

$$
h_{s}=h_{s}(D / K, R):=\#\left\{1 \leq i \leq h \mid \#\left(R_{i}^{\times}\right)=q^{s}-1\right\} .
$$

We call $h_{s}(D / K, R)$ the weight-s class number of $R$. We point out that $h_{s}$ depends only on the invariants of $R$ at finite places of $K$. The mass sum $\operatorname{Mass}(D / K, R)$ then can be written as

$$
\operatorname{Mass}(D / K, R)=\sum_{s \mid s_{0}} \frac{h_{s}}{q^{s}-1}
$$

Note that

$$
h=\sum_{s \mid s_{0}} h_{s} .
$$

For any positive divisor $s$ of $n$, we denote by $L_{s}$ the constant field extension $K \mathbb{F}_{q^{s}}$ of degree $s$. Let $O_{L_{s}}:=A \mathbb{F}_{q^{s}}$, the integral closure of $A$ in $L_{s}$. For $1 \leq i \leq h$, an optimal embedding of $O_{L_{s}}$ into $R_{i}$ is an embedding $f: L_{s} \hookrightarrow D$ such that

$$
f\left(L_{s}\right) \cap R_{i}=f\left(O_{L_{s}}\right) .
$$

If the set of optimal embeddings of $O_{L_{s}}$ into $R_{i}$ is non-empty, then there are exactly $s$ different embeddings into $R_{i}$. To see this, the map $f$ is determined by its restriction on the constant subfield $\mathbb{F}_{q^{s}}$ and the image $f\left(\mathbb{F}_{q^{s}}\right)$ is contained in the 
finite field $\mathbb{F}_{q}\left[R_{i}^{\times}\right]$. Therefore, there are exactly $s$ maps $f$. Note that an optimal embedding of $L_{s}$ into $R_{i}$ exists if and only if $\mathbb{F}_{q^{s}}$ can be embedded into $R_{i}$.

The number of optimal embeddings of $O_{L_{s}}$ into $R_{i}$ are related to the class numbers $h_{s^{\prime}}$ for $s \mid s^{\prime}$ via the following identity:

$$
\left.s \cdot \sum_{s^{\prime}: s\left|s^{\prime}\right| s_{0}} h_{s^{\prime}}=\sum_{1 \leq i \leq h} \# \text { optimal embeddings of } O_{L_{s}} \text { into } R_{i}\right\} .
$$

Let

$$
\mathbf{E}(D / K, s, R):=\sum_{1 \leq i \leq h} \#\left\{\text { optimal embeddings of } O_{L_{s}} \text { into } R_{i}\right\} .
$$

Then $\mathbf{E}(D / K, s, R)$, as the same as $h$ and $h_{s}$, also depends only on the invariants of $R$ at finite places of $K$. Suppose we can compute $\mathbf{E}(E / K, s, R)$ for each divisor $s>1$ of $s_{0}$. Then together with the mass formula (3.2) and the equation (3.5), we have enough equations to solve the numbers $h_{s}$. This gives the class number $h$ in question.

3.3. Adelization. Now we focus on the computation of $\mathbf{E}(D / K, s, R)$ for $s>1$. Fix an inclusion $\iota: L_{s_{0}}=K \mathbb{F}_{D} \hookrightarrow D$. For each positive divisor $s$ of $s_{0}$, the set of optimal embeddings of $O_{L_{s}}$ into $R_{i}$ can be identified with

$$
C_{\iota}\left(L_{s}\right)^{\times} \backslash \mathcal{E}_{\iota}\left(s, R_{i}\right) /\left(R_{i}^{\times}\right),
$$

where $C_{\iota}\left(L_{s}\right)$ is the centralizer of $\iota\left(L_{s}\right)$ in $D$ and

$$
\mathcal{E}_{\iota}\left(s, R_{i}\right):=\left\{g \in D^{\times} \mid g^{-1} \iota\left(L_{s}\right) g \cap R_{i}=g^{-1} \iota\left(O_{L_{s}}\right) g\right\} .
$$

Set

$$
\widehat{\mathcal{E}}_{\iota}(s, R):=\left\{g \in \widehat{D}^{\times} \mid \iota\left(L_{s}\right) \cap g \widehat{R} g^{-1}=\iota\left(O_{L_{s}}\right)\right\} .
$$

Then

Lemma 3.2. We have the following bijection:

$$
\begin{aligned}
\Phi: \quad \coprod_{i=1}^{h} C_{\iota}\left(L_{s}\right)^{\times} \backslash \mathcal{E}_{\iota}\left(s, R_{i}\right) / R_{i}^{\times} & \cong C_{\iota}\left(L_{s}\right)^{\times} \backslash \widehat{\mathcal{E}}_{\iota}(s, R) / \widehat{R}^{\times} \\
g \in \mathcal{E}_{\iota}\left(s, R_{i}\right) & \longmapsto
\end{aligned}
$$

where $g_{1}, \ldots, g_{h}$ are the chosen representatives of double cosets in $D^{\times} \backslash \widehat{D}^{\times} / \widehat{R}^{\times}$.

Proof. It is clear that $\Phi$ is well-defined. Now, for each $\hat{g} \in \widehat{\mathcal{E}}_{\iota}(s, R)$, there exist an element $b_{\hat{g}} \in D^{\times}$, an integer $i_{\hat{g}}$ with $1 \leq i_{\hat{g}} \leq h$, and an element $\hat{\gamma}_{\hat{g}} \in \widehat{R}^{\times}$such that

$$
\hat{g}=b_{\hat{g}} \cdot g_{i_{\hat{g}}} \cdot \hat{\gamma}_{\hat{g}}
$$

Then $b_{\hat{g}}$ must be in $\mathcal{E}_{\iota}\left(s, R_{i_{\hat{g}}}\right)$, and

$$
C_{\iota}\left(L_{s}\right)^{\times} \hat{g} \widehat{R}^{\times} \longmapsto C_{\iota}\left(L_{s}\right)^{\times} b_{\hat{g}} R_{i_{\hat{g}}}^{\times} \in C_{\iota}\left(L_{s}\right)^{\times} \backslash \mathcal{E}_{\iota}\left(s, R_{i_{\hat{g}}}\right) / R_{i_{\hat{g}}}^{\times}
$$

gives the inverse map of $\Phi$. 
Let $\widehat{L}_{s}:=L_{s} \otimes_{K} \mathbb{A}_{K}^{\infty}$, and let $C_{\iota}\left(\widehat{L}_{s}\right)$ be the centralizer of $\iota\left(\widehat{L}_{s}\right)$ in $\widehat{D}$. We have the following canonical surjective map

$$
\Psi: C_{\iota}\left(L_{s}\right)^{\times} \backslash \widehat{\mathcal{E}}_{\iota}(s, R) / \widehat{R}^{\times} \rightarrow C_{\iota}\left(\widehat{L}_{s}\right)^{\times} \backslash \widehat{\mathcal{E}}_{\iota}(s, R) / \widehat{R}^{\times} .
$$

The fiber of a double coset $C_{\iota}\left(\widehat{L}_{s}\right)^{\times} \hat{g} \widehat{R}^{\times}$under the map $\Psi$ is equal to the following double coset space

$$
C_{\iota}\left(L_{s}\right)^{\times} \backslash C_{\iota}\left(\widehat{L}_{s}\right)^{\times} /\left(C_{\iota}\left(\widehat{L}_{s}\right)^{\times} \cap \hat{g} \widehat{R}^{\times} \hat{g}^{-1}\right) .
$$

Note that the base space $C_{\iota}\left(\widehat{L}_{s}\right)^{\times} \backslash \widehat{\mathcal{E}}_{\iota}(s, R) / \widehat{R}^{\times}$can be decomposed locally:

$$
C_{\iota}\left(\widehat{L}_{s}\right)^{\times} \backslash \widehat{\mathcal{E}}_{\iota}(s, R) / \widehat{R}^{\times}=\prod_{v \neq \infty} C_{\iota}\left(L_{s, v}\right)^{\times} \backslash \mathcal{E}_{v, \iota}\left(s, R_{v}\right) / R_{v}^{\times},
$$

where

$$
L_{s, v}:=L_{s} \otimes_{K} K_{v}, \quad R_{v}:=R \otimes_{A} O_{v}, \quad O_{L_{s}, v}:=O_{L_{s}} \otimes_{A} O_{v}=\prod_{w \mid v} O_{L_{s}, w},
$$

and

$$
\mathcal{E}_{v, \iota}\left(s, R_{v}\right):=\left\{g_{v} \in D_{v}^{\times} \mid \iota\left(L_{s, v}\right) \cap g_{v} R_{v} g_{v}^{-1}=\iota\left(O_{L_{s}, v}\right)\right\} .
$$

Therefore, to compute the number of optimal embeddings in question, we need to

- give an explicit parametrization of $C_{\iota}\left(L_{s, v}\right)^{\times} \backslash \mathcal{E}_{v, \iota}\left(s, R_{v}\right) / R_{v}^{\times}$for each finite place $v$ of $K$, and

- calculate the cardinality of the fiber of each double coset in the base space $C_{\iota}\left(\widehat{L}_{s}\right)^{\times} \backslash \widehat{\mathcal{E}}_{\iota}(s, R) / \widehat{R}^{\times}$.

According to the study of local optimal embeddings in Section 7 , the double coset space $C_{\iota}\left(L_{s, v}\right)^{\times} \backslash \mathcal{E}_{v, \iota}\left(s, R_{v}\right) / R_{v}^{\times}$can be understood clearly. Besides, the fiber of a double coset in $C_{\iota}\left(\widehat{L}_{s}\right)^{\times} \backslash \widehat{\mathcal{E}}_{\iota}(s, R) / \widehat{R}^{\times}$can be identified with the set of locally free right ideal classes of a corresponding hereditary $O_{L_{s}}$-order in the centralizer $C_{\iota}\left(L_{s}\right)$. When $s>1$ and $s \mid\left[\mathbb{F}_{D}: \mathbb{F}_{q}\right]$, the algebra $C_{\iota}\left(L_{s}\right)$ is again a definite central simple algebra over $L_{s}$, with $\left[C_{\iota}\left(L_{s}\right): L_{s}\right]=(n / s)^{2}$. Repeating this process, we evaluate the class number $h(R)$ eventually.

\section{Main Results}

4.1. In this section, we analyze the number of global optimal embeddings. We establish relations among weight- $s$ class numbers $h_{s}(R)$ and those of smaller central simple subalgebras, for which we call the "generalized "transfer principle". Then we use these relations to evaluate the class number $h(R)$ recursively.

Let $\overrightarrow{\mathbf{f}}:=\left(\vec{f}_{v}\right)_{v \neq \infty}$ where for each finite place $v$ of $K, \vec{f}_{v}=\left(f_{v, 1}, \ldots, f_{v, r_{v}}\right)$ is a vector in $\mathbb{Z}_{>0}^{r_{v}}$ with $\sum_{j=1}^{r_{v}} f_{v, j}=m_{v}$, and for almost all $v$ we have $r_{v}=1$. Let $R=R(D / K, \overrightarrow{\mathbf{f}})$ be a hereditary $A$-order in $D$ so that the invariant of $R_{v}$ is $\vec{f}_{v}$ at every finite place $v$.

Let $s$ be a positive divisor of $s_{0}=\left[\mathbb{F}_{D}: \mathbb{F}_{q}\right]$. Recall the equation (3.7) (also see (3.8))

$$
s \cdot \sum_{s^{\prime}: s\left|s^{\prime}\right| s_{0}} h_{s^{\prime}}(D / K, \overrightarrow{\mathbf{f}})=\mathbf{E}(D / K, s, \overrightarrow{\mathbf{f}})
$$


where $h_{s}(D / K, \overrightarrow{\mathbf{f}})=h_{s}(D / K, R)$ and $\mathbf{E}(D / K, s, \overrightarrow{\mathbf{f}})=\mathbf{E}(D / K, s, R)$ are as in Section 3.2 ,

Before presenting our main result, we recall some notations and the invariants which we need:

- $D$ is a definite central simple algebra over $K$ with $\operatorname{dim}_{K} D=n^{2}$.

- $\kappa_{v} / d_{v} \bmod \mathbb{Z}$ is the local invariants of $D$ at $v$ and $m_{v}=n / d_{v}$.

- For each positive divisor $s$ of $n$, put

$$
\ell_{s, v}=\operatorname{gcd}(s, \operatorname{deg} v), \quad \text { and } \quad t_{s, v}=\operatorname{gcd}\left(s / \ell_{s, v}, d_{v}\right) .
$$

- We denote by $\Sigma_{L_{s}}^{0}$ the set of places $w$ of $L_{s}=K \mathbb{F}_{q^{s}}$ with $w \nmid \infty$.

Note that $\ell_{s, v}$ is the number of places of $L_{s}$ over $v$ and $t_{s, v}$ is the capacity of the central simple algebra $\Delta_{v} \otimes_{K_{v}} L_{s, w}$ over $L_{s, w}$, that is, $\Delta_{v} \otimes_{K_{v}} L_{s, w}=\operatorname{Mat}_{t_{s, v}}\left(\Delta_{w^{\prime}}\right)$.

4.2. The index set $\Omega(D / K, s, \overrightarrow{\mathbf{f}})$. Let $\overrightarrow{\mathbf{f}}=\left(\vec{f}_{v}\right)_{v \neq \infty}$ be as above, and let $s$ be a positive divisor of $s_{0}=\left[\mathbb{F}_{D}: \mathbb{F}_{q}\right]$. We define a set $\Omega(D / K, s, \overrightarrow{\mathbf{f}})$ as the product over all finite places of sets $\Omega_{v}\left(D / K, s, \vec{f}_{v}\right)$ :

$$
\Omega(D / K, s, \overrightarrow{\mathbf{f}})=\prod_{v \neq \infty} \Omega_{v}\left(D / K, s, \vec{f}_{v}\right)
$$

For each $v \in \Sigma_{K}^{0}$, let $\Omega_{v}\left(D / K, s, \overrightarrow{f_{v}}\right)$ denote the set consisting of all tuples $\left(\vec{f}_{w, *}\right)_{w \mid v}$ indexed by places $w$ of $L_{s}$ over $v$, where each $\vec{f}_{w, *}=\left(f_{w,(i, j)}\right)$ is an $r_{v} \times t_{s, v}$-matrix with non-negative integer entries $f_{w, i, j)} \in \mathbb{Z}_{\geq 0}$ (for $1 \leq i \leq r_{v}$ and $1 \leq j \leq t_{s, v}$ ), that satisfy the following conditions: If one puts

$$
f_{w, i}:=\frac{s}{\ell_{s, v} t_{s, v}} \cdot \sum_{j=1}^{t_{s, v}} f_{w,(i, j)}
$$

then

$$
\sum_{i=1}^{r_{v}} f_{w, i}=\frac{m_{v}}{\ell_{s, v}} \quad \forall w \mid v, \text { and } \sum_{w \mid v} f_{w, i}=f_{v, i} \quad \text { for } 1 \leq i \leq r_{v} .
$$

Each element of $\Omega(D / K, s, \overrightarrow{\mathbf{f}})$ is also of the form $\overrightarrow{\mathbf{f}}_{*}=\left(\vec{f}_{w, *}\right)_{w \in \Sigma_{L_{s}}^{0}}$, where $\vec{f}_{w, *}=$ $\left(f_{w,(i, j)}\right)$ with non-negative integers $f_{w, i, j)}$ that satisfy the above conditions. We put an order on the index set $\{(i, j)\}_{1 \leq i \leq r_{v}, 1 \leq j \leq t_{s, v}}$ by

$$
(i, j)<\left(i^{\prime}, j^{\prime}\right) \text { if }\left\{\begin{array}{l}
j<j^{\prime}, \text { or } \\
j=j^{\prime} \text { and } i<i^{\prime},
\end{array}\right.
$$

and write $\vec{f}_{w, *}$ as a long vector in $\mathbb{Z}_{\geq 0}^{r_{v} \cdot t_{s, v}}$, that is,

$$
\vec{f}_{w, *}=\left(f_{w,(1,1)}, \ldots, f_{w,\left(r_{v}, 1\right)}, f_{w,(1,2)}, \ldots, f_{w,\left(r_{v}, 2\right)}, \ldots, f_{w,\left(1, t_{s, v}\right)}, \ldots, f_{w,\left(r_{v}, t_{s, v}\right)}\right) .
$$

When $d_{v}=1$ and $r_{v}=1$, one gets $t_{s, v}=1$ and $\vec{f}_{v}=(n)$. In this case, we get $\vec{f}_{w, *}=(n / s)$ for all places $w$ of $L_{s}$ lying above $v$. Therefore,

$$
\#\left(\Omega_{v}\left(D / K, s, \vec{f}_{v}\right)\right)=1 .
$$

This shows that $\Omega(D / K, s, \overrightarrow{\mathbf{f}})$ is a finite set. 
Lemma 4.1. The set $\Omega_{v}\left(D / K, s, \vec{f}_{v}\right)$ is non-empty if and only if

$$
\frac{s}{\ell_{s, v} t_{s, v}} \mid f_{v, i}
$$

for $1 \leq i \leq r_{v}$.

Proof. It is clear that the non-emptiness of $\Omega_{v}\left(D / K, s, \vec{f}_{v}\right)$ implies the condition (4.4).

Note that $s / \ell_{s, v} t_{s, v}=\left[\mathbb{F}_{\Delta_{w}^{\prime}}: \mathbb{F}_{\Delta_{v}}\right]$. Since there is an embedding $L_{s, w} \hookrightarrow$ $\operatorname{Mat}_{m_{v} / \ell_{s, v}}\left(\Delta_{v}\right)$, the divisibility $\left(s / \ell_{s, v} t_{s, v}\right) \mid\left(m_{v} / \ell_{s, v}\right)$ is automatically satisfied. Conversely, suppose the condition (4.4) is satisfied. Then the set $\Omega_{v}\left(D / K, s, \vec{f}_{v}\right)$ is non-empty by the next lemma. This proves the lemma.

Lemma 4.2. Let $\left(m_{1}, \ldots, m_{\ell}\right)$ and $\left(f_{1}, \ldots, f_{r}\right)$ be two sequences of non-negative integers satisfying $\sum_{w=1}^{\ell} m_{w}=\sum_{i=1}^{r} f_{i}$. Then the set

$$
\Omega:=\left\{\left(f_{w, i}\right) \in \mathbb{Z}_{\geq 0}^{\ell} \times \mathbb{Z}_{\geq 0}^{r} \mid \sum_{w} f_{w, i}=f_{i}, \forall i, \text { and } \sum_{i} f_{w . i}=m_{w} \forall w\right\}
$$

is non-empty.

Proof. We prove this by induction on $\ell$. The statement holds clearly when $\ell=1$. For any $\ell$, choose non-negative integers $f_{\ell, 1} \ldots, f_{\ell, r}$ with $f_{\ell, i} \leq f_{i}$ for all $i=1, \ldots, r$ and $\sum_{i} f_{\ell, i}=m_{\ell}$. By induction, the set for $\left(m_{1}, \ldots, m_{\ell-1}\right)$ and $\left(f_{1}-f_{\ell, 1}, \ldots f_{r}-f_{\ell, r}\right)$ is non-empty and hence the set $\Omega$ is non-empty. This proves the lemma.

4.3. Main results. For each $\overrightarrow{\mathbf{f}}_{*} \in \Omega(D / K, s, \overrightarrow{\mathbf{f}})$, set $\overrightarrow{\mathbf{f}}_{*}^{o}:=\left(\vec{f}_{w, *}^{o}\right)_{w \in \Sigma_{L_{s}}^{0}}$ where $\vec{f}_{w, *}^{o}$ is the vector obtained by removing the zero entries of the vector $\vec{f}_{w, *}$. For example, if $\vec{f}_{w, *}=(5,0,4,1,0,1)$, then $\vec{f}_{w, *}^{o}=(5,4,1,1)$. See (3.8) for the definition of the term $\mathbf{E}(D / K, s, \overrightarrow{\mathbf{f}})$.

Theorem 4.3. Let $D$ be a definite central simple algebra of degree $n^{2}$ over $K$. For each place $v$ of $K$, the local invariant $\operatorname{inv}_{v}(D)$ is denoted by $\kappa_{v} / d_{v} \bmod \mathbb{Z}$ and $n=m_{v} d_{v}$. Let $s$ be a positive divisor of $s_{0}=\left[\mathbb{F}_{D}: \mathbb{F}_{K}\right]$ and $\overrightarrow{\mathbf{f}}=\left(\overrightarrow{f_{v}}\right)_{v \neq \infty}$, where for each finite place $v$ of $K, \vec{f}_{v}=\left(f_{v, 1}, \ldots, f_{v, r_{v}}\right)$ is a vector in $\mathbb{Z}_{>0}^{r_{v}}$ with $\sum_{i=1}^{r_{v}} f_{v, i}=m_{v}$, and for almost all $v$ we have $r_{v}=1$. Then

$$
\mathbf{E}(D / K, s, \overrightarrow{\mathbf{f}})=\sum_{\overrightarrow{\mathbf{f}}_{*} \in \Omega(D / K, s, \overrightarrow{\mathbf{f}})} h\left(D_{s}^{\prime} / L_{s}, \overrightarrow{\mathbf{f}}_{*}^{o}\right) .
$$

Here $D_{s}^{\prime}$ is the centralizer $C_{\iota}\left(L_{s}\right)$ in $D$ (for an arbitrary fixed embedding $\iota$ of $L_{s_{0}}$ to $D)$, which is a definite central simple algebra over $L_{s}$, and $h\left(D_{s}^{\prime} / L_{s}, \overrightarrow{\mathbf{f}}_{*}^{o}\right)$ is the class number of hereditary $O_{L_{s}}$ order $R\left(D_{s}^{\prime} / L_{s}, \overrightarrow{\mathbf{f}}_{*}^{o}\right)$ in $D_{s}^{\prime}$.

Proof. Fix an embedding $\iota: L_{s_{0}} \hookrightarrow D$. For any positive divisor $s$ of $s_{0}$, we must have $\ell_{s, \infty}=1$. Therefore $D_{s}^{\prime}$ is definite (with respect to the unique place of $L_{s}$ lying above $\infty$ ).

Let $R=R(D / K, \overrightarrow{\mathbf{f}})$ be a hereditary $A$-order in $D$ such that the invariant of $R$ is $\vec{f}$. By Lemma 3.2, we have

$$
\mathbf{E}(D / K, s, \overrightarrow{\mathbf{f}})=\#\left(C_{\iota}\left(L_{s}\right)^{\times} \backslash \widehat{\mathcal{E}}_{\iota}(s, R) / \widehat{R}^{\times}\right) .
$$


Consider the canonical surjective map

$$
\Psi: C_{\iota}\left(L_{s}\right)^{\times} \backslash \widehat{\mathcal{E}}_{\iota}(s, R) / \widehat{R}^{\times} \rightarrow C_{\iota}\left(\widehat{L}_{s}\right)^{\times} \backslash \widehat{\mathcal{E}}_{\iota}(s, R) / \widehat{R}^{\times} .
$$

Note that

$$
C_{\iota}\left(\widehat{L}_{s}\right)^{\times} \backslash \widehat{\mathcal{E}}_{\iota}(s, R) / \widehat{R}^{\times}=\prod_{v \neq \infty} C_{\iota}\left(L_{s, v}\right)^{\times} \backslash \mathcal{E}_{v, \iota}\left(s, R_{v}\right) / R_{v}^{\times},
$$

By Lemma 7.2. Propositions 7.5 and 7.6 (1), and Theorem 7.10 (1), we have a natural bijection

$$
C_{\iota}\left(L_{s, v}\right)^{\times} \backslash \mathcal{E}_{v, \iota}\left(s, R_{v}\right) / R_{v}^{\times} \simeq \Omega_{v}\left(D / K, s, \vec{f}_{v}\right) .
$$

From (4.1) we have a natural bijection

$$
C_{\iota}\left(\widehat{L}_{s}\right)^{\times} \backslash \widehat{\mathcal{E}}_{\iota}(s, R) / \widehat{R}^{\times} \simeq \Omega(D / K, s, \overrightarrow{\mathbf{f}}) .
$$

Let $[\hat{g}]$ be the double coset corresponding to a given $\overrightarrow{\mathbf{f}}_{*} \in \Omega(D / K, s, \overrightarrow{\mathbf{f}})$. By Propositions 7.5 7.6 (2), and Theorem 7.10 (2), we have

$$
C_{\iota}\left(L_{s}\right) \cap \hat{g} \widehat{R} \hat{g}^{-1}=R\left(D_{s}^{\prime} / L_{s}, \overrightarrow{\mathbf{f}}_{*}^{o}\right),
$$

which is a hereditary $O_{L_{s}}$-order in $D_{s}^{\prime}$. Therefore

$$
\begin{aligned}
\# \Psi^{-1}([\hat{g}]) & =\#\left(C_{\iota}\left(L_{s}\right)^{\times} \backslash C_{\iota}\left(\widehat{L}_{s}\right)^{\times} /\left(C_{\iota}\left(\widehat{L}_{s}\right)^{\times} \cap \hat{g} \widehat{R}^{\times} \hat{g}^{-1}\right)\right) \\
& =h\left(D_{s}^{\prime} / L_{s}, \overrightarrow{\mathbf{f}}_{*}^{o}\right) .
\end{aligned}
$$

This completes the proof of the theorem.

Theorem 4.4. Notations being as above. There is an optimal embedding of $O_{L_{s}}$ into a hereditary order $R^{\prime}$ in $D$ of invariant $\overrightarrow{\mathbf{f}}$ if and only if for all $v \in \Sigma_{K}^{0}$, one has

$$
\frac{s}{\ell_{s, v} t_{s, v}} \mid f_{v, i}
$$

for $1 \leq i \leq r_{v}$

Proof. This follows from (4.7) and Lemma 4.1.

It is clear that for each positive divisor $s$ of $s_{0}=\left[\mathbb{F}_{D}: \mathbb{F}_{q}\right]$, one has

$$
\left[\mathbb{F}_{D_{s}^{\prime}}: \mathbb{F}_{q^{s}}\right]=s_{0} / s
$$

Theorem 4.3 (and (3.7)) tells us that

$$
\begin{aligned}
s \cdot \sum_{s^{\prime}: s\left|s^{\prime}\right| s_{0}} h_{s^{\prime}}(D / K, \overrightarrow{\mathbf{f}}) & =\sum_{\overrightarrow{\mathbf{f}}_{*} \in \Omega(D / K, s, \overrightarrow{\mathbf{f}})} h\left(D_{s}^{\prime} / L_{s}, \overrightarrow{\mathbf{f}}_{*}^{o}\right) \\
& =\sum_{s^{\prime}: s\left|s^{\prime}\right| s_{0}}\left(\sum_{\overrightarrow{\mathbf{f}}_{*} \in \Omega(D / K, s, \overrightarrow{\mathbf{f}})} h_{s^{\prime} / s}\left(D_{s}^{\prime} / L_{s}, \overrightarrow{\mathbf{f}}_{*}^{o}\right)\right) .
\end{aligned}
$$

The following is one of main theorems of this paper, which is a refinement of the relation (4.10). 
Theorem 4.5. (The generalized transfer principle) For any two positive divisors $s$ and $s^{\prime}$ of $s_{0}$ with $s \mid s^{\prime}$, we have

$$
s \cdot h_{s^{\prime}}(D / K, \overrightarrow{\mathbf{f}})=\sum_{\overrightarrow{\mathbf{f}}_{*} \in \Omega(D / K, s, \overrightarrow{\mathbf{f}})} h_{s^{\prime} / s}\left(D_{s}^{\prime} / L_{s}, \overrightarrow{\mathbf{f}}_{*}^{o}\right) .
$$

Proof. For positive divisors $s$ and $s^{\prime}$ of $s_{0}$ with $s \mid s^{\prime}$, one observes that

$$
\Omega\left(D / K, s^{\prime}, \overrightarrow{\mathbf{f}}\right)=\coprod_{\overrightarrow{\mathbf{g}}_{*} \in \Omega(D / K, s, \overrightarrow{\mathbf{f}})} \Omega\left(D_{s}^{\prime} / L_{s}, \frac{s^{\prime}}{s}, \overrightarrow{\mathbf{g}}_{*}^{o}\right) .
$$

Suppose the statement holds in the case $s=s^{\prime}$, that is, the relation

$$
s \cdot h_{s}(D / K, \overrightarrow{\mathbf{f}})=\sum_{\overrightarrow{\mathbf{f}}_{*} \in \Omega(D / K, s, \overrightarrow{\mathbf{f}})} h_{1}\left(D_{s}^{\prime} / L_{s}, \overrightarrow{\mathbf{f}}_{*}^{o}\right)
$$

holds for any positive divisor $s$ of $s_{0}$. Then

$$
s \cdot h_{s^{\prime}}(D / K, \overrightarrow{\mathbf{f}})=\frac{s}{s^{\prime}} \cdot\left(s^{\prime} \cdot h_{s^{\prime}}(D / K, \overrightarrow{\mathbf{f}})\right)=\frac{s}{s^{\prime}} \cdot \sum_{\overrightarrow{\mathbf{f}}_{*} \in \Omega\left(D / K, s^{\prime}, \overrightarrow{\mathbf{f}}\right)} h_{1}\left(D_{s^{\prime}}^{\prime} / L_{s^{\prime}}, \overrightarrow{\mathbf{f}}_{*}^{o}\right) .
$$

On the other hand, one has

$$
\begin{aligned}
\sum_{\overrightarrow{\mathbf{g}}_{*} \in \Omega(D / K, s, \overrightarrow{\mathbf{f}})} h_{s^{\prime} / s}\left(D_{s}^{\prime} / L_{s}, \overrightarrow{\mathbf{g}}_{*}^{o}\right) \\
=\frac{s}{s^{\prime}} \sum_{\overrightarrow{\mathbf{g}}_{*} \in \Omega(D / K, s, \overrightarrow{\mathbf{f}})}\left(\sum_{\overrightarrow{\mathbf{f}}_{*} \in \Omega\left(D_{s}^{\prime} / L_{s}, s^{\prime} / s, \overrightarrow{\mathbf{g}}_{*}^{o}\right)} h_{1}\left(D_{s^{\prime}}^{\prime} / L_{s^{\prime}}, \overrightarrow{\mathbf{f}}_{*}^{o}\right)\right) .
\end{aligned}
$$

Therefore to complete the proof, it suffices to prove the equality (4.12). We prove this by induction on the number $\mu(D / K, s):=\sum_{i} n_{i}$, where $\left[\mathbb{F}_{D}: \mathbb{F}_{K}\right] / s=\prod_{i} p_{i}^{n_{i}}$ is the prime decomposition.

Note that $\left[\mathbb{F}_{D_{s}^{\prime}}: \mathbb{F}_{L_{s}}\right]=s_{0} / s$ for any positive divisor $s$ of $s_{0}=\left[\mathbb{F}_{D}: \mathbb{F}_{K}\right]$. Therefore (4.12) holds for $\mu(D / K, s)=0$, i.e. $s=s_{0}=\left[\mathbb{F}_{D}: \mathbb{F}_{K}\right]$. Indeed, by Theorem 4.3 we have

$$
\begin{aligned}
s_{0} \cdot h_{s_{0}}(D / K, \overrightarrow{\mathbf{f}}) & =\mathbf{E}\left(D / K, s_{0}, \overrightarrow{\mathbf{f}}\right) \\
& =\sum_{\overrightarrow{\mathbf{f}}_{*} \in \Omega\left(D / K, s_{0}, \overrightarrow{\mathbf{f}}\right)} h\left(D_{s_{0}}^{\prime} / L_{s_{0}}, \overrightarrow{\mathbf{f}}_{*}^{o}\right) \\
& =\sum_{\overrightarrow{\mathbf{f}}_{*} \in \Omega\left(D / K, s_{0}, \overrightarrow{\mathbf{f}}\right)} h_{1}\left(D_{s_{0}}^{\prime} / L_{s_{0}}, \overrightarrow{\mathbf{f}}_{*}^{o}\right) .
\end{aligned}
$$

Suppose the equality (4.12) holds for any pair $\left(D^{\prime \prime} / K^{\prime \prime}, s^{\prime \prime}\right)$ with

$$
\mu\left(D^{\prime \prime} / K^{\prime \prime}, s^{\prime \prime}\right)<\mu(D / K, s) .
$$

Then we get

$$
\begin{aligned}
\mathbf{E}(D / K, s, \overrightarrow{\mathbf{f}}) & =s \cdot \sum_{s^{\prime}: s\left|s^{\prime}\right| s_{0}} h_{s^{\prime}}(D / K, \overrightarrow{\mathbf{f}}) \\
& =s \cdot h_{s}(D / K, \overrightarrow{\mathbf{f}})+\sum_{\substack{s^{\prime}: s\left|s^{\prime}\right| s_{0} \\
s<s^{\prime}}} \frac{s}{s^{\prime}}\left(s^{\prime} \cdot h_{s^{\prime}}(D / K, \overrightarrow{\mathbf{f}})\right) .
\end{aligned}
$$


Since $\mu\left(D / K, s^{\prime}\right)<\mu(D / K, s)$ for any positive divisor $s^{\prime}$ of $s_{0}$ with $s \mid s^{\prime}$ and $s<s^{\prime}$, by induction hypothesis, one has

$$
\sum_{\substack{s^{\prime}: s\left|s^{\prime}\right| s_{0} \\ s<s^{\prime}}} \frac{s}{s^{\prime}}\left(s^{\prime} \cdot h_{s^{\prime}}(D / K, \overrightarrow{\mathbf{f}})\right)=\sum_{\substack{s^{\prime}: s\left|s^{\prime}\right| s_{0} \\ s<s^{\prime}}} \frac{s}{s^{\prime}}\left(\sum_{\substack{\vec{f}_{*} \in \Omega\left(D / K, s^{\prime}, \overrightarrow{\mathbf{f}}\right)\\}} h_{1}\left(D_{s^{\prime}}^{\prime} / L_{s^{\prime}}, \overrightarrow{\mathbf{f}}_{*}^{O}\right)\right) .
$$

On the other hand, by Theorem 4.3 we get

$$
\begin{aligned}
& \mathbf{E}(D / K, s, \overrightarrow{\mathbf{f}}) \\
= & \sum_{\overrightarrow{\mathbf{f}}_{*} \in \Omega(D / K, s, \overrightarrow{\mathbf{f}})}\left(\sum_{s^{\prime}: s\left|s^{\prime}\right| s_{0}} h_{s^{\prime} / s}\left(D_{s}^{\prime} / L_{s}, \overrightarrow{\mathbf{f}}_{*}^{o}\right)\right) \\
= & \sum_{\overrightarrow{\mathbf{f}}_{*} \in \Omega(D / K, s, \overrightarrow{\mathbf{f}})} h_{1}\left(D_{s}^{\prime} / L_{s}, \overrightarrow{\mathbf{f}}_{*}^{o}\right)+\sum_{\substack{s^{\prime}: s\left|s^{\prime}\right| s_{0}, s<s^{\prime}}}\left(\sum_{\overrightarrow{\mathbf{g}}_{*} \in \Omega(D / K, s, \overrightarrow{\mathbf{f}})} h_{s^{\prime} / s}\left(D_{s}^{\prime} / L_{s}, \overrightarrow{\mathbf{g}}_{*}^{o}\right)\right) .
\end{aligned}
$$

Since $\mu\left(D_{s}^{\prime} / L_{s}, s^{\prime} / s\right)<\mu(D / K, s)$ for any positive divisor $s^{\prime}$ of $s_{0}$ with $s \mid s^{\prime}$ and $s<s^{\prime}$, by induction hypothesis again we obtain that

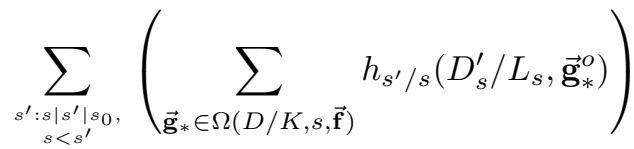

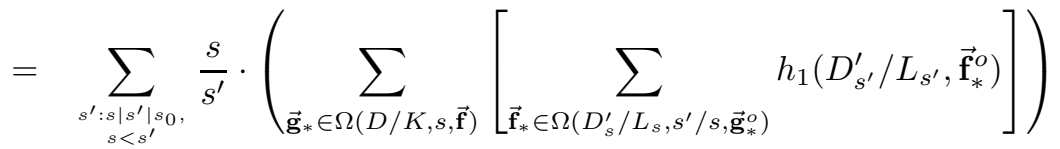

$$
\begin{aligned}
& =\sum_{\substack{s^{\prime}: s\left|s^{\prime}\right| s_{0} \\
s<s^{\prime}}} \frac{s}{s^{\prime}}\left(\sum_{\substack{\overrightarrow{\mathbf{f}}_{*} \in \Omega\left(D / K, s^{\prime}, \overrightarrow{\mathbf{f}}\right)\\
}} h_{1}\left(D_{s^{\prime}}^{\prime} / L_{s^{\prime}}, \overrightarrow{\mathbf{f}}_{*}^{O}\right)\right) \text {. }
\end{aligned}
$$

Then the equation (4.12) follows from (4.13) - (4.16). This completes the proof of the theorem.

Recall that

$$
\operatorname{Mass}(D / K, \overrightarrow{\mathbf{f}})=\sum_{s \mid\left[\mathbb{F}_{D}: \mathbb{F}_{K}\right]} \frac{h_{s}(D / K, \overrightarrow{\mathbf{f}})}{q^{s}-1} .
$$

Theorem 4.5 states that one can compute the weight- $s^{\prime}$ class number $h_{s^{\prime}}(D / K, \overrightarrow{\mathbf{f}})$ in terms of the weight- $s^{\prime} / s$ class numbers of a central simple algebra $D_{s}^{\prime} / L_{s}$ of smaller degree. Then by induction one can compute the weight- $s^{\prime}$ class number $h_{s^{\prime}}(D / K, \overrightarrow{\mathbf{f}})$. However, since the index set $\Omega(D / K, s, \overrightarrow{\mathbf{f}})$ could be quite large, the computation by induction would be very complicated. The following theorem states that we can compute directly a modified version of the right hand side of the equation (4.11) in Theorem 4.5 without going through the induction step, but keep the same information so that we can compute the weight- $s^{\prime}$ class number $h_{s^{\prime}}(D / K, \overrightarrow{\mathbf{f}})$. This simplifies the computation significantly. 
Theorem 4.6. Notations being as above, one has

$$
s \cdot \sum_{s^{\prime}: s\left|s^{\prime}\right|\left[\mathbb{F}_{D}: \mathbb{F}_{K}\right]} \frac{h_{s^{\prime}}(D / K, \overrightarrow{\mathbf{f}})}{q^{s^{\prime}}-1}=\sum_{\overrightarrow{\mathbf{f}}_{*} \in \Omega(D / K, s, \overrightarrow{\mathbf{f}})} \operatorname{Mass}\left(D_{s}^{\prime} / L_{s}, \overrightarrow{\mathbf{f}}_{*}^{o}\right) .
$$

Proof. This is obtained by multiplying the factor $\left(q^{s^{\prime}}-1\right)^{-1}$ on (4.11) and summing over all positive integers $s^{\prime}$ with $s\left|s^{\prime}\right| s_{0}$.

Set $\operatorname{Mass}(D / K):=\operatorname{Mass}\left(D / K, \overrightarrow{\mathbf{f}}_{\max }\right)$ where $\overrightarrow{\mathbf{f}}_{\max }$ is the invariant of a maximal $A$-order in $D$. Then the mass formula in Theorem 3.1 says that

$$
\operatorname{Mass}(D / K, \overrightarrow{\mathbf{f}})=\operatorname{Mass}(D / K) \cdot \prod_{v \in \Sigma_{K}^{0}} \mathcal{T}^{\prime}{ }_{v}\left(D / K, \vec{f}_{v}\right),
$$

where for a vector $\vec{h}_{v}=\left(h_{v, 1}, \ldots, h_{v, r_{v}}\right) \in \mathbb{Z}_{\geq 0}^{r_{v}}$, we set

$$
\mathcal{T}^{\prime}{ }_{v}\left(D / K, \vec{h}_{v}\right):=\frac{\prod_{i=1}^{m_{v}}\left(N(v)^{d_{v} i}-1\right)}{\prod_{i=1}^{r_{v}}\left(\prod_{j=1}^{h_{v, i}}\left(N(v)^{d_{v} j}-1\right)\right)} .
$$

For each finite place $v$ of $K$, define

$$
\Theta_{v}\left(D / K, s, \vec{f}_{v}\right):=\sum_{\left(\vec{f}_{w, *}\right)_{w \mid v} \in \Omega_{v}\left(D / K, s, \vec{f}_{v}\right)}\left(\prod_{w \mid v} \mathcal{T}^{\prime}{ }_{w}\left(D_{s}^{\prime} / L_{s}, \vec{f}_{w, *}\right)\right) .
$$

It is clear that $\Theta_{v}\left(D / K, s, \vec{f}_{v}\right)=1$ if $d_{v}=1$ and $\vec{f}_{v}=(n)$. Therefore we can rewrite Theorem 4.6 as the following theorem, which reduces the computation in purely local terms.

Theorem 4.7. Notations being as above, one has

$$
s . \sum_{s^{\prime}: s\left|s^{\prime}\right|\left[\mathbb{F}_{D}: \mathbb{F}_{K}\right]} \frac{h_{s^{\prime}}(D / K, \overrightarrow{\mathbf{f}})}{q^{s^{\prime}}-1}=\operatorname{Mass}\left(D_{s}^{\prime} / L_{s}\right) \cdot \prod_{v \in \Sigma_{K}^{0}} \Theta_{v}\left(D / K, s, \vec{f}_{v}\right) .
$$

4.4. Explicit computation of $\Theta_{v}\left(D / K, s, \vec{f}_{v}\right)$. In this subsection we give a simple method to compute the term $\Theta_{v}\left(D / K, s, \vec{f}_{v}\right)$ effectively.

Fix a finite place $v$ of $K$. Recall that $D_{v}=D \otimes_{K} K_{v} \cong \operatorname{Mat}_{m_{v}}\left(\Delta_{v}\right)$ and $\Delta_{v}$ is a central division algebra over $K_{v}$ with $\left[\Delta_{v}: K_{v}\right]=d_{v}^{2}$. For any positive divisor $s$ of $\left[\mathbb{F}_{D}: \mathbb{F}_{K}\right]$, recall $\ell_{s, v}:=\operatorname{gcd}(s, \operatorname{deg} v)$ and $t_{s, v}:=\operatorname{gcd}\left(s / \ell_{s, v}, d_{v}\right)$. We have $L_{s, v}=\prod_{w=1}^{\ell_{s, v}} L_{w}$, where each $L_{w}$ is a unramified extension over $K_{v}$ of degree $s / \ell_{s, v}$, and $\Delta_{v} \otimes_{K_{v}} L_{w} \simeq \operatorname{Mat}_{t_{s, v}}\left(\Delta_{w}^{\prime}\right)$. The division algebra $\Delta_{w}^{\prime}$ has degree $d_{v}^{\prime}:=d_{v} / t_{s, v}$ over $L_{w}$ and its residue field $\mathbb{F}_{\Delta_{w}^{\prime}}$ has cardinality $N(v)^{d_{v}^{\prime} s / \ell_{s, v}}$.

We assume that the set $\Omega_{v}\left(D / K, s, \vec{f}_{v}\right)$ is non-empty. By Lemma 4.1, this is equivalent to the condition

$$
\frac{s}{\ell_{s, v} t_{s, v}} \mid f_{v, i}, \quad \forall 1 \leq i \leq r_{v} .
$$

Since $s$ divides $\left[\mathbb{F}_{D}: \mathbb{F}_{K}\right]$, one gets the divisibility

$$
\frac{s}{\ell_{s, v} t_{s, v}} \mid\left(\frac{m_{v}}{\ell_{s, v}}\right) \text {. }
$$


The completion $D_{s}^{\prime} \otimes_{L_{s}} L_{s, v}$ of the division algebra $D_{s}^{\prime}$ at $v$ is the centralizer of $L_{s, v}$ in $D_{v}$, which is isomorphic to

$$
\prod_{w=1}^{\ell_{s, v}} \operatorname{Mat}_{m_{v}^{(s)}}\left(\Delta_{w}^{\prime}\right)
$$

where

$$
m_{v}^{(s)}=\left(\frac{s}{\ell_{s, v} t_{s, v}}\right)^{-1} \cdot \frac{m_{v}}{\ell_{s, v}}=\frac{m_{v} t_{s, v}}{s} .
$$

Set

$$
f_{v, i}^{(s)}:=\left(\frac{s}{\ell_{s, v} t_{s, v}}\right)^{-1} \cdot f_{v, i} \in \mathbb{N}, \quad \text { for } 1 \leq i \leq r_{v}
$$

Then the set $\Omega_{v}:=\Omega_{v}\left(D / K, s, \vec{f}_{v}\right)$ consists of all elements

$$
\left(f_{w,(i, j)}\right)_{w, i, j} \in \mathbb{Z}_{\geq 0}^{\ell_{s, v}} \times \mathbb{Z}_{\geq 0}^{r_{v}} \times \mathbb{Z}_{\geq 0}^{t_{s, v}}
$$

that satisfy the following conditions

$$
\sum_{i, j} f_{w,(i, j)}=m_{v}^{(s)}, \forall 1 \leq w \leq \ell_{s, v} \quad \text { and } \quad \sum_{w, j} f_{w,(i, j)}=f_{v, i}^{(s)}, \forall 1 \leq i \leq r_{v} .
$$

Consider the following formal power series

$$
F(T):=1+a_{1} T+a_{2} T^{2}+\cdots+a_{\nu} T^{\nu}+\cdots \in \mathbb{Q}[[T]],
$$

where

$$
a_{\nu}:=\prod_{k=1}^{\nu}\left[N(v)^{\left(d_{v}^{\prime} s / \ell_{v, s}\right) \cdot k}-1\right]^{-1}
$$

Put

$$
G(\underline{X}, \underline{Y}, \underline{Z}):=\prod_{w=1}^{\ell_{s, v}} \prod_{i=1}^{r_{v}} \prod_{j=1}^{t_{s, v}} F\left(X_{w} \cdot Y_{i} \cdot Z_{j}\right) \in \mathbb{Q}[[\underline{X}, \underline{Y}, \underline{Z}]]
$$

where $\underline{X}=\left(X_{1}, \ldots, X_{\ell_{s, v}}\right), \underline{Y}=\left(Y_{1}, \ldots, Y_{r_{v}}\right)$, and $\underline{Z}=\left(Z_{1}, \ldots, Z_{t_{s, v}}\right)$. Then we can use the generating function $G$ to compute the term $\Theta_{v}\left(D / K, s, \vec{f}_{v}\right)$.

Proposition 4.8. The coefficient of the monomial

$$
X_{1}^{m_{v}^{(s)}} \cdots X_{\ell_{s, v}}^{m_{v}^{(s)}} Y_{1}^{f_{v, 1}^{(s)}} \cdots Y_{r_{v}}^{f_{v, r_{v}}^{(s)}}
$$

of the formal power series $G(\underline{X}, \underline{Y}, 1, \ldots, 1) \in \mathbb{Q}[[\underline{X}, \underline{Y}]]$ is equal to

$$
\left[\prod_{k=1}^{m_{v}^{(s)}}\left[N(v)^{\left(d_{v}^{\prime} s / \ell_{v, s}\right) \cdot k}-1\right]\right]^{-\ell_{s, v}} \cdot \Theta_{v}\left(D / K, s, \vec{f}_{v}\right) .
$$

Proof. First for each $w=1, \ldots, \ell_{s, v}$,

$$
D_{s}^{\prime} \otimes_{L_{s}} L_{w} \cong \operatorname{Mat}_{m_{v}^{(s)}}\left(\Delta_{w}^{\prime}\right)
$$


where $\Delta_{w}^{\prime}$ is a central division algebra over $L_{w}$ of degree $d_{v}^{\prime}$. Using the definition of $\Theta_{v}$ we can express the term $\Theta_{v}\left(D / K, s, \vec{f}_{v}\right)$ as follows

$$
\sum_{\left(f_{w,(i, j)}\right) \in \Omega_{v}}\left[\prod_{w=1}^{\ell_{s, v}}\left(\frac{\prod_{k=1}^{m_{v}^{(s)}}\left[N(v)^{\left(d_{v}^{\prime} s / \ell_{v, s}\right) \cdot k}-1\right]}{\prod_{i=1}^{r_{v}} \prod_{j=1}^{t_{s, v}} \prod_{k,(i, j)}\left[N(v)^{\left(d_{v}^{\prime} s / \ell_{v, s}\right) \cdot k}-1\right]}\right] .\right.
$$

Therefore the term

$$
\left[\prod_{k=1}^{m_{v}^{(s)}}\left[N(v)^{\left(d_{v}^{\prime} s / \ell_{v, s}\right) \cdot k}-1\right]\right]^{-\ell_{s, v}} \cdot \Theta_{v}\left(D / K, s, \vec{f}_{v}\right)
$$

is equal to

$$
\sum_{\left(f_{w,(i, j)}\right)_{w, i, j}, \text { with [4.22) }}\left[\prod_{w=1}^{\ell_{s, v}} \prod_{i=1}^{r_{v}} \prod_{j=1}^{t_{s, v}} a_{f_{w,(i, j)}}\right] .
$$

On the other hand, one sees that the coefficient of the monomial

$$
X_{1}^{m_{v}^{(s)}} \cdots X_{\ell_{s, v}}^{m_{v}^{(s)}} Y_{1}^{f_{v, 1}^{(s)}} \cdots Y_{r_{v}}^{f_{v, r}^{(s)}}
$$

of the formal power series $G(\underline{X}, \underline{Z}, 1, \ldots, 1)$ is equal to

$$
\sum_{\left(f_{w, i, j}\right)_{w, i, j} \text { with 44.22) }}\left[\prod_{w=1}^{\ell_{s, v}} \prod_{i=1}^{r_{v}} \prod_{j=1}^{t_{s, v}} a_{f_{w, i, j}}\right] .
$$

The result follows from the equations (4.24) and (4.25). This completes the proof of the proposition.

4.5. A recursive formula for computing class numbers. In this subsection, we present an explicit recursive formula to compute the class numbers $h_{s}(D / K, \overrightarrow{\mathbf{f}})$ in terms of mass sums. Recall that $\operatorname{Mass}(D / K, \overrightarrow{\mathbf{f}})$ can be expressed in terms of special zeta values using the mass formula (Theorem 3.1). Then the target class number $h(D / K, \overrightarrow{\mathbf{f}})$ is simply the following sum

$$
h(D / K, \overrightarrow{\mathbf{f}})=\sum_{s \in \mathbb{N},} h_{s \mid\left[\mathbb{F}_{D}: \mathbb{F}_{K}\right]} h_{s}(D / K, \overrightarrow{\mathbf{f}}) .
$$

We recall that $(2.2) \mathbb{F}_{D}\left(\right.$ resp. $\left.\mathbb{F}_{K}\right)$ is the constant field of $D$ (resp. $K$ ). One computes the degree $s_{0}=\left[\mathbb{F}_{D}: \mathbb{F}_{K}\right]$ using Lemma 2.3.

For each positive divisor $s$ of $\left[\mathbb{F}_{D}: \mathbb{F}_{K}\right]$, we call the number of prime factors of the integer $\left[\mathbb{F}_{D}: \mathbb{F}_{K}\right] / s$ with multiplicity the depth of $(D / K, s)$, and denote it by $\mu(D / K, s)$. That is, if $\left[\mathbb{F}_{D}: \mathbb{F}_{K}\right] / s=\prod_{i} p_{i}^{n_{i}}$ is the prime decomposition, then $\mu(D / K, s)=\sum_{i} n_{i}$. 
(1) The case $\mu(D / K, s)=0$, i.e. $s=s_{0}$. It is clear that $\left[\mathbb{F}_{D_{s}^{\prime}}: \mathbb{F}_{L_{s}}\right]=1$. Theorems 4.6 and 4.7 states that

$$
\begin{aligned}
h_{s}(D / K, \overrightarrow{\mathbf{f}}) & =\frac{q^{s}-1}{s} \cdot \sum_{\overrightarrow{\mathbf{f}}_{*} \in \Omega(D / K, s, \overrightarrow{\mathbf{f}})} \operatorname{Mass}\left(D_{s}^{\prime} / L_{s}, \overrightarrow{\mathbf{f}}_{*}^{o}\right) \\
& =\frac{q^{s}-1}{s} \cdot \operatorname{Mass}\left(D_{s}^{\prime} / L_{s}\right) \cdot \prod_{v \in \Sigma_{K}^{0}} \Theta_{v}\left(D / K, s, \vec{f}_{v}\right) .
\end{aligned}
$$

Therefore we have evaluated the class number $h_{s}(D / K, \overrightarrow{\mathbf{f}})$ when $\mu(D / K, s)=1$.

(2) Given a positive integer $N$, assume that the class number $h_{s^{\prime}}(D / K, \overrightarrow{\mathbf{f}})$ has been evaluated for any positive integer $s^{\prime}$ with $\mu\left(D / K, s^{\prime}\right) \leq N$. Let $s$ be a positive divisor of $\left[\mathbb{F}_{D}: \mathbb{F}_{K}\right]$ with the depth $\mu(D / K, s)=N+1$. Theorem 4.7 says that

$$
s \cdot \sum_{s^{\prime}: s\left|s^{\prime}\right|\left[\mathbb{F}_{D}: \mathbb{F}_{K}\right]} \frac{h_{s^{\prime}}(D / K, \overrightarrow{\mathbf{f}})}{q^{s^{\prime}}-1}=\operatorname{Mass}\left(D_{s}^{\prime} / L_{s}\right) \cdot \prod_{v \in \Sigma_{K}^{0}} \Theta_{v}\left(D / K, s, \vec{f}_{v}\right) .
$$

Since the right hand side of the equation (4.27) can be computed explicitly, one evaluates the term $h_{s}(D / K, \overrightarrow{\mathbf{f}})$ by

$$
\begin{aligned}
h_{s}(D / K, \overrightarrow{\mathbf{f}})= & \frac{q^{s}-1}{s} \cdot\left(\operatorname{Mass}\left(D_{s}^{\prime} / L_{s}\right) \cdot \prod_{v \in \Sigma_{K}^{0}} \Theta_{v}\left(D / K, s, \vec{f}_{v}\right)\right. \\
& \left.-s \cdot \sum_{\substack{s^{\prime}: s\left|s^{\prime}\right|\left[\mathbb{P}_{D}: \mathbb{F}_{K}\right], s^{\prime}>s}} \frac{h_{s^{\prime}}(D / K, \overrightarrow{\mathbf{f}})}{q^{s^{\prime}}-1}\right) .
\end{aligned}
$$

By the steps (1) and (2), we can compute all class numbers $h_{s}(D / K, \overrightarrow{\mathbf{f}})$ and hence compute the desired class number $h(D / K, \overrightarrow{\mathbf{f}})$.

Remark 4.9. Since the mass sum can be expressed by integral values of the zeta function, the class number $h(D / K, \overrightarrow{\mathbf{f}})$ can be expressed in terms of special zeta values eventually.

\section{SPECIAL CASES}

In this section, we explain first that Theorem 4.5 is a generalization of Gekeler's transfer principle [7. We also deduce an explicit class number formula in the case where the degree $s_{0}=\left[\mathbb{F}_{D}: \mathbb{F}_{K}\right]$ is a prime. Then we give one example to illustrate how to compute the class number using the results in Section 4 .

5.1. Gekeler's transfer principle. Let $K, \infty, A$ be as before and $v_{0}$ a fixed finite place of $K$. For any positive integer $n$, let $\Lambda\left(K, \infty, v_{0} ; n\right)$ denote the set of isomorphism classes of supersingular Drinfeld $A$-modules of rank $n$ over $\overline{\mathbb{F}}_{v_{0}}$. For any object $\phi$ in $\Lambda\left(K, \infty, v_{0} ; n\right)$, the automorphism group $\operatorname{Aut}(\phi)$ of $\phi$ is isomorphic to $\mathbb{F}_{q^{s}}^{\times}$for some positive integer $s$ with $s \mid n$. For any positive divisor $s$ of $n$, let

$$
\Lambda\left(K, \infty, v_{0} ; n, s\right):=\left\{\phi \in \Lambda\left(K, \infty, v_{0} ; n\right) \mid \operatorname{Aut}(\phi) \simeq \mathbb{F}_{q^{s}}^{\times}\right\} .
$$

Theorem 5.1 (The transfer principle [7). For any two positive divisors $s$ and $s^{\prime}$ of $n$ with $s \mid s^{\prime}$, there is a natural bijection

$$
\Phi: \Lambda\left(L_{s}, \infty_{s}, v_{0, s} ; n / s^{\prime}, s^{\prime} / s\right) \stackrel{\sim}{\longrightarrow} \Lambda\left(K, \infty, v_{0} ; n, s^{\prime}\right),
$$


where $L_{s}$ is the constant field extension of degree $s, v_{0, s}$ and $\infty_{s}$ are the unique places of $L_{s}$ over $v_{0}$ and $\infty$, respectively. Recall that $O_{L_{s}}$ is the integral closure of $A$ in $L_{s}$. The map $\Phi$ sends a Drinfeld $O_{L_{s}}$-module $\phi_{s}: O_{L_{s}} \rightarrow \overline{\mathbb{F}}_{v_{0, s}}\{\tau\}$ to the Drinfeld A-module $\phi:=\left.\phi_{s}\right|_{A}$.

Let $D$ be the endomorphism algebra $\operatorname{End}(\phi) \otimes_{A} K$ of $\phi$, where $\phi$ is an object in $\Lambda\left(K, \infty, v_{0} ; n\right)$. It is a basic fact that (cf. [6])

$$
-\operatorname{inv}_{\infty}(D) \equiv \operatorname{inv}_{v_{0}}(D) \equiv 1 / n \bmod \mathbb{Z}
$$

and

$$
\operatorname{inv}_{v}(D) \equiv 0 \bmod \mathbb{Z} \text { for } v \neq v_{0}, \infty .
$$

The subset $\Lambda\left(K, \infty, v_{0} ; n, s\right)$ is non-empty if and only if $s \mid s_{0}$, where $s_{0}:=\left[\mathbb{F}_{D}: \mathbb{F}_{K}\right]$. Using the notation as before, $m_{\infty}=m_{v_{0}}=1$. By Lemma 2.1, the integer $s_{0}$ is the largest divisor $s$ of $n$ that satisfies $(\operatorname{deg} \infty, s)=\left(\operatorname{deg} v_{0}, s\right)=1$. It follows that

$$
s_{0}=\prod_{p \nmid \operatorname{deg} v_{0} \cdot \operatorname{deg} \infty} p^{\operatorname{ord}_{p}(n)} .
$$

Let $R:=\operatorname{End}(\phi)$ be the endomorphism ring of $\phi$, where $\phi \in \Lambda\left(K, \infty, v_{0} ; n\right)$. Then $R$ is a maximal $A$-order in $D$ and $h(D / K, R)=\# \Lambda\left(K, \infty, v_{0} ; n\right)$. Moreover, for any positive divisor $s$ of $s_{0}$ one gets

$$
h_{s}(D / K, R)=\# \Lambda\left(K, \infty, v_{0} ; n, s\right) .
$$

Therefore Gekeler's transfer principle asserts that for $s\left|s^{\prime}\right| s_{0}$, one has

$$
h_{s^{\prime}}(D / K, R)=h_{s^{\prime} / s}\left(D_{s}^{\prime} / L_{s}, R_{s}\right)
$$

where $R_{s}$ is a maximal $O_{L_{s}}$-order in $D_{s}^{\prime}$.

Now, since $R$ is a maximal $A$-order of $D$, the invariant $\overrightarrow{\mathbf{f}}_{R}=\left(\vec{f}_{v}\right)_{v \neq \infty}$ of $R$ is as follows

$$
r_{v}=1 \text { and } \overrightarrow{f_{v}}=\left(m_{v}\right), \quad \forall v \in \Sigma_{K}^{0} .
$$

As $D$ is of Drinfeld type, we have

$$
m_{v}=\left\{\begin{array}{ll}
1 & \text { if } v=\infty \text { or } v_{0}, \\
n & \text { otherwise }
\end{array} \text { and } d_{v}= \begin{cases}n & \text { if } v=\infty \text { or } v_{0} \\
1 & \text { otherwise }\end{cases}\right.
$$

Consider elements $\overrightarrow{\mathbf{f}}_{*}=\left(\vec{f}_{w, *}\right)_{w \in \Sigma_{L_{s}}^{0}}$ in $\Omega\left(D / K, s, \overrightarrow{\mathbf{f}}_{R}\right)$. For $w \neq v_{0, s}$, the only possible choice for $\vec{f}_{w, *}$ for $w \neq v_{0, s}$ is $(n / s)$. For $w=v_{0, s}$, the vector $\vec{f}_{w, *}=$ $\left(f_{w,(1, j)}\right)_{1 \leq j \leq s}$ satisfies the following property:

$$
f_{w,(1, j)}=1 \text { for some } j \text { and } f_{w,\left(1, j^{\prime}\right)}=0 \text { for } j^{\prime} \neq j \text {. }
$$

Therefore:

(i) The cardinality of $\Omega\left(D / K, s, \overrightarrow{\mathbf{f}}_{R}\right)$ is $s$.

(ii) For any $\overrightarrow{\mathbf{f}}_{*} \in \Omega\left(D / K, s, \overrightarrow{\mathbf{f}}_{R}\right)$, the ring $R\left(D_{s}^{\prime} / L_{s}, \overrightarrow{\mathbf{f}}_{*}^{o}\right)$ is a maximal $O_{L_{s}}$-order $R_{s}$ in $D_{s}^{\prime}$.

By Theorem 4.5, we have

$$
\begin{aligned}
s \cdot h_{s^{\prime}}(D / K, R) & =s \cdot h_{s^{\prime}}\left(D / K, \overrightarrow{\mathbf{f}}_{R}\right) \\
& =\sum_{\overrightarrow{\mathbf{f}}_{*} \in \Omega\left(D / K, s, \overrightarrow{\mathbf{f}}_{R}\right)} h_{s^{\prime} / s}\left(D_{s}^{\prime} / L_{s}, \overrightarrow{\mathbf{f}}_{*}^{o}\right) \\
& =s \cdot h_{s^{\prime} / s}\left(D_{s}^{\prime} / L_{s}, R_{s}\right) .
\end{aligned}
$$


This is exactly Gekeler's transfer principle.

5.2. The case where $\left[\mathbb{F}_{D}: \mathbb{F}_{K}\right]$ is a prime. Assume that $s_{0}=\left[\mathbb{F}_{D}: \mathbb{F}_{K}\right]$ is a prime number. For any hereditary $A$-order $R(D / K, \overrightarrow{\mathbf{f}})$, one has

$$
h(D / K, \overrightarrow{\mathbf{f}})=h_{1}(D / K, \overrightarrow{\mathbf{f}})+h_{s_{0}}(D / K, \overrightarrow{\mathbf{f}}),
$$

and

$$
\operatorname{Mass}(D / K, \overrightarrow{\mathbf{f}})=\frac{h_{1}(D / K, \overrightarrow{\mathbf{f}})}{q-1}+\frac{h_{s_{0}}(D / K, \overrightarrow{\mathbf{f}})}{q^{s_{0}}-1} .
$$

By Theorem 4.7, we have

$$
h_{s_{0}}(D / K, \overrightarrow{\mathbf{f}})=\frac{q^{s_{0}}-1}{s_{0}} \cdot \sum_{\overrightarrow{\mathbf{f}}_{*} \in \Omega\left(D / K, s_{0}, \overrightarrow{\mathbf{f}}\right)} \operatorname{Mass}\left(D_{s_{0}}^{\prime} / L_{s_{0}}, \overrightarrow{\mathbf{f}}_{*}^{o}\right) .
$$

Therefore we get the following result.

Theorem 5.2. When $s_{0}=\left[\mathbb{F}_{D}: \mathbb{F}_{K}\right]$ is a prime number, we have

$$
\begin{aligned}
h(D / K, \overrightarrow{\mathbf{f}})= & (q-1) \cdot \operatorname{Mass}(D / K, \overrightarrow{\mathbf{f}}) \\
& +\frac{q^{s_{0}}-q}{s_{0}} \cdot \operatorname{Mass}\left(D_{s_{0}}^{\prime} / L_{s_{0}}\right) \cdot \prod_{v \in \Sigma_{K}^{0}} \Theta_{v}\left(D / K, s_{0}, \overrightarrow{f_{v}}\right) .
\end{aligned}
$$

Now, suppose $n$ is a prime number (where $[D: K]=n^{2}$ ). For each place $v$ of $K$, let

$$
\epsilon(v):= \begin{cases}1 & \text { if } n \nmid \operatorname{deg} v \\ 0 & \text { otherwise. }\end{cases}
$$

There exists an embedding of $L_{n}$ into $D$ if and only if $\prod_{v \in S_{D}} \epsilon(v)=1$. Since $D_{n}^{\prime} \cong L_{n}$ and $A \mathbb{F}_{q^{n}}=O_{L_{n}}$, we have

$$
\operatorname{Mass}\left(D_{n}^{\prime} / L_{n}\right)=\frac{\#\left(\operatorname{Pic}\left(O_{L_{n}}\right)\right)}{\left(q^{n}-1\right)}
$$

and

$$
\Theta_{v}\left(D / K, n, \overrightarrow{f_{v}}\right)=\#\left(\Omega_{v}\left(D / K, n, \vec{f}_{v}\right)\right) .
$$

Therefore it suffices to compute $\#\left(\Omega_{v}\left(D / K, n, \vec{f}_{v}\right)\right)$ for the place $v$ where $D_{v}$ is a division algebra or where the order $R_{v}$ is not maximal.

Let $S^{\prime}$ be the set of finite places of $K$ where $R$ is not maximal. Since $n$ is a prime, the intersection of $S_{D}$ and $S^{\prime}$ is empty. Suppose $v \in S_{D}-\{\infty\}$. For $\left(\vec{f}_{w, *}\right)_{w \mid v} \in \Omega_{v}\left(D / K, n, \vec{f}_{v}\right)$, there are precisely $n$ choices for $\vec{f}_{w, *}$ if $w$ is the only one place lying above $v$ where $v \in S_{D}-\{\infty\}$, i.e.

$$
\#\left(\Omega_{v}\left(D / K, n, \vec{f}_{v}\right)\right)=n .
$$

Let $v \in S^{\prime}$. If $\ell_{n, v}=\operatorname{gcd}(n, \operatorname{deg} v)=1$, then $\Omega_{v}\left(D / K, n, \vec{f}_{v}\right)$ is empty. Suppose $\ell_{n, v}=n$ for all $v \in S^{\prime}$. Then $t_{n, v}=1$ and $m_{v} / \ell_{n, v}=1$ for $v \in S^{\prime}$. Fix a place $v \in S^{\prime}$. Then for places $w_{1}, \ldots, w_{n}$ lying above $v$, one has

$$
\vec{f}_{w_{\nu}, *}=\left(f_{w_{\nu},(i, 1)}\right)_{1 \leq i \leq r_{v}}
$$

with $f_{w_{\nu},(i, 1)}=1$ for some $i$ and $f_{w_{\nu},\left(i^{\prime}, 1\right)}=0$ for $i^{\prime} \neq i$, and

$$
\sum_{\nu=1}^{n} f_{w_{\nu},(i, 1)}=f_{v, i} \text { for } 1 \leq i \leq r_{v}
$$


WEI AND YU

Therefore the number of choices of $\left(\vec{f}_{w_{1}, *}, \ldots, \vec{f}_{w_{n}, *}\right) \in \Omega_{v}\left(D / K, n, \vec{f}_{v}\right)$, i.e. the cardinality of $\Omega_{v}\left(D / K, n, \overrightarrow{f_{v}}\right)$, is

$$
\frac{n !}{f_{v, 1} ! \cdots f_{v, r_{v}} !} \text {. }
$$

We conclude that $\Omega(D / K, n, \overrightarrow{\mathbf{f}})$ is non-empty if and only if

$$
\prod_{v \in S_{D}} \epsilon(v) \cdot \prod_{v \in S^{\prime}}(1-\epsilon(v))=1
$$

and in this case, one has

$$
\#(\Omega(D / K, n, \overrightarrow{\mathbf{f}}))=\prod_{v \in S-\{\infty\}} n \cdot \prod_{v \in S^{\prime}} \frac{n !}{f_{v, 1} ! \cdots f_{v, r_{v}} !} .
$$

By Theorem 5.2 and the relations (5.4)-(5.9), we obtain the following result.

Theorem 5.3. Let $D$ be a definite central simple algebra over $K$ (with respect to $\infty)$ of degree $n$. Let $R=R(D / K, \overrightarrow{\mathbf{f}})$ be a hereditary A-order in $D$ with invariant $\overrightarrow{\mathbf{f}}$. Assume that $n$ is a prime number. Let $S$ be the set of places where $D$ is ramified (including $\infty$ ), and $S^{\prime}$ be the set of finite places where the hereditary $A$-order $R$ is not maximal. Then the class number $h(D / K, \overrightarrow{\mathbf{f}})$ is equal to

$$
\begin{aligned}
& (q-1) \cdot \operatorname{Mass}(D / K, \overrightarrow{\mathbf{f}}) \\
+ & \frac{q^{n}-q}{q^{n}-1} \cdot \frac{\# \operatorname{Pic}\left(O_{L_{n}}\right)}{n^{2}} \cdot \prod_{v \in S}(n \cdot \epsilon(v)) \cdot \prod_{v \in S^{\prime}}\left(\frac{n !}{f_{v, 1} ! \cdots f_{v, r_{v}} !}(1-\epsilon(v))\right) .
\end{aligned}
$$

Theorem 5.3 agrees with the main results of Denert and Van Geel 4, Theorems 3 and 9] when $K$ is the rational function field. We remark that the proof of Theorem 5.3 does not rely on the Eichler-Brandt trace formula.

5.3. Example. Here we present one explicit example to show how to compute the class number by the recursive formula in 4.5 .

Let $K=\mathbb{F}_{q}(T)$ with $q=3$ and $A=\mathbb{F}_{q}[T]$. Let $D$ be the central division algebra over $K$ with

$$
\begin{gathered}
-\operatorname{inv}_{\infty}(D)=\operatorname{inv}_{T}(D)=\frac{1}{4} \bmod \mathbb{Z} \\
\operatorname{inv}_{T+1}(D)=\operatorname{inv}_{T+2}(D)=\frac{1}{2} \bmod \mathbb{Z}
\end{gathered}
$$

and $\operatorname{inv}_{v}(D)=0 \bmod \mathbb{Z}$ for $v \neq \infty, T, T+1, T+2$. Let $R$ be a maximal $A$-order in $D$. Then the invariant $\overrightarrow{\mathbf{f}}=\left(\vec{f}_{v}\right)_{v \neq \infty}$ of $R$ is:

$$
\begin{aligned}
\vec{f}_{T} & =(1) \\
\vec{f}_{T+1} & =(2) \\
\vec{f}_{T+2} & =(2) \\
\vec{f}_{v} & =(4) \text { for } v \neq T, T+1, T+2 .
\end{aligned}
$$

By Lemma 2.3, we have $\left[\mathbb{F}_{D}: \mathbb{F}_{K}\right]=4$. It suffices to compute $h_{1}(D / K, \overrightarrow{\mathbf{f}})$, $h_{2}(D / K, \overrightarrow{\mathbf{f}})$, and $h_{4}(D / K, \overrightarrow{\mathbf{f}})$. 
Note that $L_{4}=\mathbb{F}_{q^{4}}(T), O_{L_{4}}=\mathbb{F}_{q^{4}}[T]$, and $D_{4}^{\prime} \cong L_{4}$. By Theorem 4.7.

$$
\begin{aligned}
h_{4}(D / K, \overrightarrow{\mathbf{f}}) & =\frac{q^{4}-1}{4} \cdot \operatorname{Mass}\left(D_{4}^{\prime} / L_{4}\right) \cdot \prod_{0 \leq i \leq 2} \Theta_{T+i}\left(D / K, 4, \vec{f}_{T+i}\right) \\
& =\frac{1}{4} \cdot \prod_{0 \leq i \leq 2} \Theta_{T+i}\left(D / K, 4, \vec{f}_{T+i}\right) .
\end{aligned}
$$

Since $\operatorname{deg} T=\operatorname{deg} T+1=\operatorname{deg} T+2=1$, there exists only one place $w_{T}$ (resp. $w_{T+1}, w_{T+2}$ ) of $L_{4}$ lying above $T$ (resp. $T+1, T+2$ ). Therefore

$$
\ell_{4, T}=\ell_{4, T+1}=\ell_{4, T+2}=1 \text {, }
$$

and

$$
t_{4, T}=4, t_{4, T+1}=t_{4, T+2}=2 .
$$

This also tells us that

$$
\Theta_{T+i}\left(D / K, 4, \vec{f}_{T+i}\right)=\#\left(\Omega_{T+i}\left(D / K, 4, \vec{f}_{T+i}\right)\right) .
$$

When $w=w_{T}$, for any $\vec{f}_{w, *}=\left(f_{w,(1, j)}\right)_{1 \leq j \leq 4} \in \Omega_{T}\left(D / K, 4, \vec{f}_{T}\right)$, one has

$$
\sum_{j=1}^{4} f_{w,(1, j)}=1 \text {. }
$$

This means that there are 4 choices for $\vec{f}_{w_{T}, *}$, i.e.

$$
\#\left(\Omega_{T}\left(D / K, 4, \vec{f}_{T}\right)\right)=4 \text {. }
$$

When $w=w_{T+1}$ or $w_{T+2}$, any vector $\vec{f}_{w, *}=\left(f_{w,(1, j)}\right)_{1 \leq j \leq 2}$ satisfies that

$$
\frac{4}{1 \cdot 2} \cdot \sum_{j=1}^{2} f_{w,(1, j)}=2 \text {. }
$$

Therefore there are 2 choices for $\vec{f}_{w_{T+1}, *}$ and $\vec{f}_{w_{T+2}, *}$, i.e.

$$
\#\left(\Omega_{T+1}\left(D / K, 4, \vec{f}_{T+1}\right)\right)=\#\left(\Omega_{T+2}\left(D / K, 4, \vec{f}_{T+2}\right)\right)=2 .
$$

We then conclude that

$$
h_{4}(D / K, \overrightarrow{\mathbf{f}})=4
$$

Next, we compute $h_{2}(D / K, \overrightarrow{\mathbf{f}})$. It is clear that there exists only one place $w_{T}$ (resp. $w_{T+1}, w_{T+2}$ ) of $L_{2}$ lying above $T$ (resp. $T+1, T+2$ ), and

$$
\ell_{2, T}=\ell_{2, T+1}=\ell_{2, T+2}=1, t_{2, T}=t_{2, T+1}=t_{2, T+2}=2 .
$$

By Theorem 4.7, we have

$$
\frac{h_{2}(D / K, \overrightarrow{\mathbf{f}})}{q^{2}-1}+\frac{h_{4}(D / K, \overrightarrow{\mathbf{f}})}{q^{4}-1}=\frac{1}{2} \cdot \operatorname{Mass}\left(D_{2}^{\prime} / L_{2}\right) \cdot \prod_{0 \leq i \leq 2} \Theta_{T+i}\left(D / K, 2, \vec{f}_{T+i}\right) .
$$

The mass formula in Theorem 3.1 says that

$$
\operatorname{Mass}\left(D_{2}^{\prime} / L_{2}\right)=\frac{1}{q^{4}-1}=\frac{1}{80}
$$

It remains to compute $\Theta_{T+i}\left(D / K, 2, \vec{f}_{T+i}\right)$ for $0 \leq i \leq 2$. 
The vectors $\vec{f}_{w_{T, *}}=\left(f_{w_{T},(1, j)}\right)_{1 \leq j \leq 2} \in \Omega_{T}\left(D / K, 2, \vec{f}_{T}\right)$ satisfy that

$$
\sum_{j=1}^{2} f_{w_{T},(1, j)}=1 \text {; }
$$

the vectors $\vec{f}_{w_{T+1}, *}=\left(f_{w_{T+1},(1, j)}\right)_{1 \leq j \leq 2} \in \Omega_{T+1}\left(D / K, 2, \vec{f}_{T+1}\right)$ and the vectors $\vec{f}_{w_{T+2}, *}=\left(f_{w_{T+2},(1, j)}\right)_{1 \leq j \leq 2} \in \Omega_{T+1}\left(D / K, 2, \vec{f}_{T+1}\right)$ satisfy that

$$
\sum_{j=1}^{2} f_{w_{T+1},(1, j)}=2=\sum_{j=1}^{2} f_{w_{T+2},(1, j)} .
$$

This means that

$$
\begin{aligned}
\vec{f}_{w_{T}, *} & =(1,0) \text { or }(0,1), \\
\vec{f}_{w_{T+1}, *} & =(2,0) \text { or }(1,1) \text { or }(0,2), \\
\vec{f}_{w_{T+2}, *} & =(2,0) \text { or }(1,1) \text { or }(0,2) .
\end{aligned}
$$

Hence

$$
\Theta_{T}\left(D / K, 2, \vec{f}_{T}\right)=2
$$

and

$$
\Theta_{T+1}\left(D / K, 2, \vec{f}_{T+1}\right)=\Theta_{T+2}\left(D / K, 2, \vec{f}_{T+2}\right)=2+\frac{q^{4}-1}{q^{2}-1}=12 .
$$

Therefore we get

$$
h_{2}(D / K, \overrightarrow{\mathbf{f}})=14 .
$$

Recall

$$
\frac{h_{1}(D / K, \overrightarrow{\mathbf{f}})}{q-1}+\frac{h_{2}(D / K, \overrightarrow{\mathbf{f}})}{q^{2}-1}+\frac{h_{4}(D / K, \overrightarrow{\mathbf{f}})}{q^{4}-1}=\operatorname{Mass}(D / K, \overrightarrow{\mathbf{f}}) .
$$

From the mass formula in Theorem 3.1, we have

$$
\operatorname{Mass}(D / K, \overrightarrow{\mathbf{f}})=169 / 5 .
$$

Together with the equations (5.11) and (5.12), we finally get

$$
h_{1}(D / K, \overrightarrow{\mathbf{f}})=64 \text { and } h(D / K, \overrightarrow{\mathbf{f}})=82 .
$$

\section{NON-PRINCIPAL GENERA}

In the previous sections, we study the class number $h(R)$ of locally free right ideal classes of $R$ (the principal genus). In this section, we study the class number of ideal classes of $R$ which are not necessarily locally free. We show that the class number of any genus of ideal classes of $R$ can be computed in terms of the that of locally free ideal classes of another order $R^{\prime}$, which is still hereditary. As a result, our previous results on the computation of the class number of the locally free ideal classes of an arbitrary hereditary order $R$ can be extended to that of arbitrary ideal classes of an arbitrary hereditary order (in $D$ ).

Keep the notation as before, in particular $K, \infty, A, D, R$ have the same meaning in the previous sections, By a right ideal $I$ of $R$ we mean an $A$-lattice $I$ in $D$ which is also a right $R$-submodule of $D$. Recall that a genus of right $R$-ideals is a maximal set of right $R$-ideals in $D$ where any two ideals are mutually equivalent locally 
everywhere, that is, for two ideals $I$ and $J$, there exists an element $\alpha_{v} \in D_{v}^{\times}$such that $J_{v}=\alpha_{v} \cdot I_{v}$ for any finite place $v$ of $K$. Two ideals $I$ and $J$ in a genus $\mathcal{L}$ are said to be globally equivalent if there is an element $\alpha \in D^{\times}$such that $J=\alpha I$. Let $\mathcal{L} / \sim$ denote the set of globally equivalent classes in the genus $\mathcal{L}$ and $h(\mathcal{L}):=\#(\mathcal{L} / \sim)$, the cardinality of $\mathcal{L} / \sim$, called the class number of $\mathcal{L}$. The set of locally free $R$-ideals forms a genus, which is called the principal genus; the others are called non-principal genera.

Let $v$ be a finite place of $K$. Suppose the invariant of $R_{v}$ is $\mathbf{f}_{v}=\left(f_{v, 1}, \ldots, f_{v, r_{v}}\right)$. Then it is known that (cf. [1] (39.23) Theorem) any indecomposable $R_{v}$-module is one of the form

$$
M_{1}, M_{2}, \ldots, M_{r_{v}}
$$

where $M_{1}=O_{\Delta_{v}}^{m_{v}}, M_{i}=M_{i-1} \cdot \operatorname{rad}\left(R_{v}\right)$ for $1<i \leq r_{v}$, and $\operatorname{rad}\left(R_{v}\right)$ is the Jacobson radical of $R_{v}$. Recall that $D_{v} \simeq \operatorname{Mat}_{m_{v}}\left(\Delta_{v}\right)$, where $\Delta_{v}$ is the division part and $O_{\Delta_{v}}$ is the maximal order in $\Delta_{v}$. Suppose a right ideal $I$ of $R$ is given. Then as an $R_{v}$-module, $I_{v}$ must be isomorphic to

$$
M_{1}^{g_{v, 1}} \oplus M_{2}^{g_{v, 2}} \oplus \cdots \oplus M_{r_{v}}^{g_{v, r_{v}}}
$$

for some $\mathbf{g}_{v}=\left(g_{v, 1}, \ldots, g_{v, r_{v}}\right)$ with $g_{v, i} \in \mathbb{Z}_{\geq 0}$ for each $i$ and $\sum_{i=1}^{r_{v}} g_{v, i}=m_{v}$. The datum $\mathbf{g}_{v}$ fixes an isomorphism class of right $R_{v}$-ideals, and we call it the isomorphism type of $I_{v}$ or the local isomorphism type of $I$ at $v$. Therefore we conclude the following result.

Proposition 6.1. The set of all genera of right $R$-ideals can be parametrized by the vectors $\mathbf{g}:=\left(\mathbf{g}_{v}\right)_{v \neq \infty}$, where

$$
\mathbf{g}_{v}=\left(g_{v, 1}, \ldots, g_{v, r_{v}}\right) \in \mathbb{Z}_{\geq 0}^{r_{v}} \text { with } \sum_{i=1}^{r_{v}} g_{v, i}=m_{v}
$$

and $r_{v}$ is the period of $R_{v}$. More precisely, let $I(\mathbf{g})$ be the ideal of $R$ such that for all finite places $v$

$$
I(\mathbf{g})_{v}=M_{1}^{g_{v, 1}} \oplus M_{2}^{g_{v, 2}} \oplus \cdots \oplus M_{r_{v}}^{g_{v, r}} .
$$

Then for any ideal $J$ of $R$, there exists a unique vector $\mathbf{g}$ such that $J$ and $I(\mathbf{g})$ are in the same genus.

Remark. When $R_{v}$ is a maximal order, we have $r_{v}=1$ and so $I_{v}$ must be isomorphic to $R_{v}$ as $R_{v}$-module. Therefore there are only finitely many genera of $R$.

Let $I$ be a right ideal of $R$, and let $\mathcal{L}(I)$ be the genus of right $R$-ideals that contains $I$. Since any member $J \in \mathcal{L}(I)$ has the property $J_{v}=\alpha_{v} I_{v}$, it follows that

$$
\mathcal{L}(I) \cong \widehat{D}^{\times} / \widehat{R}_{I}^{\times}
$$

where $R_{I}$ is the left order of $I$. Therefore,

$$
\mathcal{L}(I) / \sim \cong D^{\times} \backslash \widehat{D}^{\times} / \widehat{R}_{I}^{\times} \cong \mathrm{Cl}\left(R_{I}\right)
$$

and hence one gets $h(\mathcal{L}(I))=h\left(R_{I}\right)$, the class number of locally free right $R$-ideal classes.

We now describe $R_{I}$. We may assume that $I=I(\mathbf{g})$ for some vector $\mathbf{g}$ as in (6.2). For each $\mathbf{g}_{v}$ we define another vector $\mathbf{g}_{v}^{o}$ by removing the zero entries of $\mathbf{g}_{v}$. For example if $\mathbf{g}_{v}=(3,6,0,1,0)$ (with local period $r_{v}=5$ ), then we define the vector $\mathbf{g}_{v}^{o}$ to be $(3,6,1)$ (with new local period $r_{v}^{\prime}=3$ ). 
Proposition 6.2. For each finite place $v$ of $K$, we have

$$
R_{I(\mathbf{g}), v}=R_{I(\mathbf{g})} \otimes_{A} O_{v} \cong \operatorname{Mat}\left(\mathbf{g}_{v}^{o}, O_{\Delta_{v}}\right) .
$$

In particular, $R_{I(\mathbf{g})}$ is a hereditary A-order in $D$ whose local invariant at $v$ is equal to $\mathbf{g}_{v}^{o}$.

Proof. Under the identification of $D_{v}$ and $\operatorname{Mat}_{m_{v}}\left(\Delta_{v}\right), I(\mathbf{g})_{v}$ consists of elements $\left(Y_{i, j}\right)_{1 \leq i, j \leq r_{v}}$ satisfying that

$$
Y_{i, j} \in \begin{cases}\operatorname{Mat}_{g_{v, i} \times f_{v, j}}\left(O_{\Delta_{v}}\right) & \text { if } i \leq j, \\ \operatorname{Mat}_{g_{v, i} \times f_{v, j}}\left(\mathfrak{P}_{v}\right) & \text { if } i>j .\end{cases}
$$

Let $\left(Z_{i, j}\right)_{1 \leq i, j \leq r_{v}}$ be an element in $D_{v}$ where $Z_{i, j} \in \operatorname{Mat}_{g_{i} \times g_{j}}\left(\Delta_{v}\right)$. Then $\left(Z_{i, j}\right)_{1 \leq i, j \leq r_{v}}$ is in $R_{I(\mathbf{g}), v}$ if and only if for any $\left(Y_{i, j}\right)_{1 \leq i, j \leq r_{v}} \in I(\mathbf{g})_{v}$,

$$
\sum_{k=1}^{r_{v}} Z_{i, k} \cdot Y_{k, j} \in \begin{cases}\operatorname{Mat}_{g_{v, i} \times f_{v, j}}\left(O_{\Delta_{v}}\right) & \text { if } i \leq j, \\ \operatorname{Mat}_{g_{v, i} \times f_{v, j}}\left(\mathfrak{P}_{v}\right) & \text { if } i>j .\end{cases}
$$

For $1 \leq k \leq r_{v}$, plugging elements $\left(Y_{i, j}\right)_{1 \leq i, j \leq r_{v}}$ of $I(\mathbf{g})_{v}$ with $Y_{i, j}=0$ if $i \neq k$ in (6.4), we get

$$
Z_{i, k} \cdot Y_{k, j} \in \begin{cases}\operatorname{Mat}_{g_{v, i} \times f_{v, j}}\left(O_{\Delta_{v}}\right) & \text { if } i \leq j \\ \operatorname{Mat}_{g_{v, i} \times f_{v, j}}\left(\mathfrak{P}_{v}\right) & \text { if } i>j .\end{cases}
$$

This implies that $\left(Z_{i, j}\right)_{1 \leq i, j \leq r_{v}}$ is in $R_{I(\mathbf{g}), v}$ if and only if

$$
Z_{i, j} \in \begin{cases}\operatorname{Mat}_{g_{v} \times g_{v, j}}\left(O_{\Delta_{v}}\right) & \text { if } i \leq j, \\ \operatorname{Mat}_{g_{v, i} \times g_{v, j}}\left(\mathfrak{P}_{v}\right) & \text { if } i>j .\end{cases}
$$

Therefore $R_{I(\mathbf{g}), v}$ is a hereditary $O_{v}$-order with invariant $\mathbf{g}_{v}^{o}$.

Together with the parametrization of the genera of $R$-ideals in Proposition 6.1 we obtain the following result.

Theorem 6.3. The total class number of right ideal classes of $R$ is equal to

$$
\sum_{\mathbf{g}} h(\mathbf{g})
$$

where $\mathbf{g}$ runs through the vectors described in Proposition 6.1 and $h(\mathbf{g}):=h\left(R_{I(\mathbf{g})}\right)$.

Remark. After computing the class number of $R_{I(\mathbf{g})}$ for each $\mathbf{g}$, we obtain the total class number of right ideal classes of $R$.

\section{LOCAL OPTIMAL EMBEDDINGS}

7.1. Fix a non-Archimedean local field $F$. Let $D$ be a finite dimensional central simple algebra over $F$. Take any simple left $D$-submodule $V$ of $D$. Let $\Delta=$ $\left(\operatorname{End}_{D}(V)\right)^{\mathrm{op}}$, which is a central division algebra over $F$. Then $V$ can be viewed as a free right $\Delta$-module, and $D$ is canonically isomorphic to $\operatorname{End}_{\Delta}(V)$. Let $m:=$ $\operatorname{rank}_{\Delta}(V)$. Then

$$
D \cong \operatorname{Mat}_{m}(\Delta) .
$$

Let $d$ be the positive integer such that $d^{2}=[\Delta: F]$. For any field extension $F^{\prime}$ over $F$, there exists an embedding of $F^{\prime}$ into $D$ if and only if $\left[F^{\prime}: F\right]$ divides $n:=m d$. In general, let

$$
L=L_{1} \times L_{2} \times \cdots \times L_{\ell}
$$


where for $1 \leq w \leq \ell, L_{w}$ is a finite field extension of $F$. Then

Lemma 7.1. (1) There exists an $F$-algebra embedding $\iota: L \hookrightarrow D \cong \operatorname{End}_{\Delta}(V)$ if and only if there exists positive integers $m_{1}, \ldots, m_{\ell}$ such that

$$
m_{1}+\cdots+m_{\ell}=m=\operatorname{rank}_{\Delta}(V) \text { and }\left[L_{w}: F\right] \mid m_{w} d .
$$

(2) Given two embeddings $\iota_{1}$ and $\iota_{2}$ of $L$ into D. There exists an element $g$ in $D^{\times}$ such that

$$
\iota_{1}(\alpha)=g^{-1} \iota_{2}(\alpha) g, \forall \alpha \in L
$$

if and only if $\operatorname{rank}_{\Delta}\left(\iota_{1}\left(e_{w}\right) V\right)=\operatorname{rank}_{\Delta}\left(\iota_{2}\left(e_{w}\right) V\right)$ for $1 \leq w \leq \ell$, where $e_{w}$ is the idempotent in $L$ corresponding to $L_{w}$.

Proof. Suppose there exists an $F$-algebra embedding $\iota: L \hookrightarrow \operatorname{End}_{\Delta}(V)$. For $1 \leq w \leq \ell$, let $e_{w}$ be the idempotent in $L$ corresponding to $L_{w}$. Then $\iota\left(e_{w}\right) V \neq 0$ and $\iota$ induces an embedding of $L_{w}$ into $\operatorname{End}_{\Delta}\left(\iota\left(e_{w}\right) V\right)$. Set $m_{w}:=\operatorname{rank}_{\Delta}\left(\iota\left(e_{w}\right) V\right)$ for each $w$. We have

$$
m_{1}+\cdots+m_{\ell}=m \text { and }\left[L_{w}: F\right] \mid m_{w} d .
$$

Conversely, suppose we can find positive integers $m_{1}, \ldots, m_{\ell}$ satisfying the desired property. Consider any decomposition

$$
V=V_{1} \oplus V_{2} \oplus \cdots \oplus V_{\ell}
$$

with $\operatorname{rank}_{\Delta}\left(V_{w}\right)=m_{w}$. There exists $\iota_{w}: L_{w} \hookrightarrow \operatorname{End}_{\Delta}\left(V_{w}\right)$ for $1 \leq w \leq \ell$. Then the composition of $\iota_{1} \times \cdots \times \iota_{\ell}$ and the natural embedding of $\prod_{w=1}^{\ell} \operatorname{End}_{\Delta}\left(V_{w}\right)$ into $\operatorname{End}_{\Delta}(V)$ gives an embedding $\iota: L \hookrightarrow D$. Therefore the proof of (1) is complete.

For (2), if there exists $g \in D^{\times}$such that

$$
\iota_{1}(\alpha)=g^{-1} \iota_{2}(\alpha) g
$$

then for $1 \leq w \leq \ell$,

$$
\operatorname{rank}_{\Delta}\left(\iota_{1}\left(e_{w}\right) V\right)=\operatorname{rank}_{\Delta}\left(g^{-1} \iota_{2}\left(e_{w}\right) V\right)=\operatorname{rank}_{\Delta}\left(\iota_{2}\left(e_{w}\right) V\right) .
$$

Conversely, suppose $\operatorname{rank}_{\Delta}\left(\iota_{1}\left(e_{w}\right) V\right)=\operatorname{rank}_{\Delta}\left(\iota_{2}\left(e_{w}\right) V\right)$ for $1 \leq w \leq \ell$. Then $\iota_{1}\left(e_{w}\right) V$ and $\iota_{2}\left(e_{w}\right) V$ are isomorphic as $\left(L_{w}, \Delta\right)$-bimodules since $\Delta \otimes_{F} L_{w}$ is a central simple algebra over $L_{w}$. Let $g_{w}: \iota_{1}\left(e_{w}\right) V \stackrel{\sim}{\longrightarrow} \iota_{2}\left(e_{w}\right) V$ be an isomorphism of $\left(L_{w}, \Delta\right)$-bimodules. Then the element $g$ in $D^{\times}$corresponding to the automorphism

$$
g_{1} \times \cdots \times g_{\ell}: V=\bigoplus_{w=1}^{\ell} \iota_{1}\left(e_{w}\right) V \rightarrow \bigoplus_{w=1}^{\ell} \iota_{2}\left(e_{w}\right) V=V
$$

satisfies that

$$
\iota_{1}(\alpha)=g^{-1} \iota_{2}(\alpha) g, \forall \alpha \in L .
$$

This completes the proof of (2).

The vector $\left(m_{1}, \ldots, m_{\ell}\right)$ where $m_{w}=\operatorname{rank}_{\Delta}\left(\iota\left(e_{w}\right) V\right)$ is called the type of the embedding $\iota: L \hookrightarrow D$.

Now, we fix an $F$-algebra embedding $\iota: L=L_{1} \times \cdots \times L_{\ell} \hookrightarrow D$ of type $\left(m_{1}, \ldots, m_{\ell}\right)$. Denote by $O_{F}$ (resp. $\left.O_{L_{w}}\right)$ the valuation ring of $F\left(\right.$ resp. $\left.L_{w}\right)$ and set

$$
O_{L}:=O_{L_{1}} \times \cdots \times O_{L_{\ell}} .
$$

For any $O_{F}$-order $R$ of $D$, we call $\iota$ an optimal embedding of $O_{L}$ into $R$ if

$$
\iota(L) \cap R=\iota\left(O_{L}\right) .
$$


Two optimal embeddings $\iota_{1}$ and $\iota_{2}$ of the same type are called equivalent modulo $R^{\times}$if there exists an element $u \in R^{\times}$such that

$$
\iota_{1}(\alpha)=u \cdot \iota_{2}(\alpha) \cdot u^{-1}, \forall \alpha \in L .
$$

Then Lemma 7.1 implies that

Lemma 7.2. The set of equivalence classes of optimal embeddings of type $\left(m_{1}, \ldots m_{\ell}\right)$ from $O_{L}$ into $R$ modulo $R^{\times}$can be identified with

$$
C_{\iota}(L)^{\times} \backslash \mathcal{E}_{\iota}(R) / R^{\times}
$$

where $C_{\iota}(L)$ is the centralizers of $\iota(L)$ in $D$ and

$$
\mathcal{E}_{\iota}(R):=\left\{g \in D^{\times}: \iota(L) \cap g R g^{-1}=\iota\left(O_{L}\right)\right\} .
$$

Suppose $R$ is hereditary, i.e. any right (or equivalently left) ideal of $R$ is projective as an $R$-module. Let $O_{\Delta}$ be the maximal compact subring of $\Delta$ and $\mathfrak{P}_{\Delta}$ be the maximal two-sided ideal of $O_{\Delta}$. Then there exists a vector $\vec{f}_{R}=\left(f_{1}, \ldots, f_{r}\right) \in \mathbb{Z}_{>0}^{r}$ such that

$$
R \cong \operatorname{Mat}_{m}\left(\vec{f}_{R}, O_{\Delta}\right)
$$

where the ring $\operatorname{Mat}_{m}\left(\vec{f}_{R}, O_{\Delta}\right)$ consists of elements $\left(X_{i, j}\right)_{1 \leq i, j \leq r}$ in $\operatorname{Mat}_{m}\left(O_{\Delta}\right)$ such that

$$
X_{i, j} \in \begin{cases}\operatorname{Mat}_{f_{i} \times f_{j}}\left(O_{\Delta}\right) & \text { if } i \leq j, \\ \operatorname{Mat}_{f_{i} \times f_{j}}\left(\mathfrak{P}_{\Delta}\right) & \text { if } i>j .\end{cases}
$$

The number $r$ is called the period of $R$; the vector $\vec{f}_{R}:=\left(f_{1}, \ldots, f_{r}\right)$ is called the invariant of $R$, which is uniquely determined by $R$ up to cyclic permutations. When $r=m$ and $f_{i}=1$ for all $i$, the order $R$ is an Iwahori order, which is conjugate to the preimage of the set of upper triangular matrices over $\mathbb{F}_{\Delta}:=O_{\Delta} / \mathfrak{P}_{\Delta}$. Next, we connect optimal embeddings of a given type from $O_{L}$ into a hereditary $O_{F}$-order $R$ of $D$ modulo $R^{\times}$with the "flags" of $\left(O_{L}, O_{\Delta}\right)$-bimodules.

7.2. $\left(O_{L}, O_{\Delta}\right)$-flags. Recall that $D \cong \operatorname{End}_{\Delta}(V)$ and $V$ is a free right $\Delta$-module of $\operatorname{rank}_{\Delta}(V)=m$.

Definition 7.3. Let $N_{1}, \ldots, N_{r}$ be free right $O_{\Delta}$-modules in $V$ of $O_{\Delta}$-rank $m$. We call $N_{*}:=\left(N_{1}, \ldots, N_{r}\right)$ an $O_{\Delta}$-flag in $V$ of type $\vec{f}=\left(f_{1}, \ldots, f_{r}\right) \in \mathbb{Z}_{>0}^{r}$ if

(1) $N_{1} \supsetneq N_{2} \supsetneq \cdots \supsetneq N_{r} \supsetneq N_{r+1}:=N_{1} \mathfrak{P}_{\Delta}$.

(2) $\operatorname{dim}_{\mathbb{F}_{\Delta}}\left(N_{i} / N_{i+1}\right)=f_{i}$ for $1 \leq i \leq r$.

The set $\operatorname{Hom}_{O_{\Delta}}\left(N_{*}, N_{*}^{\prime}\right)$ of morphisms of $O_{\Delta}$-flags in $V$ of type $\vec{f}$ consists of endomorphisms $\phi \in \operatorname{End}_{\Delta}(V)$ such that $\phi\left(N_{i}\right) \subseteq N_{i}^{\prime}$ for $1 \leq i \leq r$.

Fix a $\Delta$-basis $\left\{x_{1}, \ldots, x_{m}\right\}$ of $V$. Given a vector $\vec{f}=\left(f_{1}, \ldots, f_{r}\right) \in \mathbb{Z}_{>0}^{r}$, set

$$
f_{(i)}:=\sum_{j=1}^{i} f_{j} \quad \text { for } 1 \leq i \leq r, \text { and } f_{(0)}:=0 .
$$

Then we have a strictly increasing sequence of integers $0=f_{(0)}<f_{(1)}<\cdots<$ $f_{(r)}=m$. For $1 \leq i \leq r$, let

$$
M_{i}:=\left(\bigoplus_{k=1}^{m-f_{(i-1)}} x_{k} O_{\Delta}\right) \oplus\left(\bigoplus_{k=m-f_{(i-1)}+1}^{m} x_{k} \mathfrak{P}_{\Delta}\right) .
$$


Then $M_{*}(\vec{f}):=\left(M_{1}, \ldots, M_{r}\right)$ is an $O_{\Delta}$-flag in $V$ of type $\vec{f}$, and

$$
\operatorname{End}_{O_{\Delta}}\left(M_{*}(\vec{f})\right) \cong \operatorname{Mat}_{m}\left(\vec{f}, O_{\Delta}\right)
$$

It is clear that every $O_{\Delta}$-flag in $V$ of type $\vec{f}$ is of the form

$$
g M_{*}(\vec{f}):=\left(g M_{1}, \ldots, g M_{r}\right)
$$

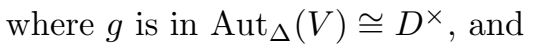

$$
\operatorname{End}_{O_{\Delta}}\left(g M_{*}(\vec{f})\right)=g \operatorname{End}_{O_{\Delta}}\left(M_{*}(\vec{f})\right) g^{-1} .
$$

Suppose an $F$-algebra embedding $\iota: L \hookrightarrow D$ of type $\left(m_{1}, \ldots, m_{\ell}\right)$ is given.

Definition 7.4. Let $N_{*}=\left(N_{1}, \ldots, N_{r}\right)$ be an $O_{\Delta}$-flag in $V$ of type $\vec{f}$. We call $N_{*}$ an $\left(O_{L}, O_{\Delta}\right)$-flag in $V$ of type $(\iota, \vec{f})$ if

$$
\iota\left(O_{L}\right) \cdot N_{i} \subseteq N_{i} \text { for all } 1 \leq i \leq r
$$

The set $\operatorname{Hom}_{\left(O_{L}, O_{\Delta}\right)}\left(N_{*}, N_{*}^{\prime}\right)$ of morphisms of $\left(O_{L}, O_{\Delta}\right)$-flags in $V$ of type $(\iota, \vec{f})$ consists of morphisms in $\operatorname{Hom}_{O_{\Delta}}\left(N_{*}, N_{*}^{\prime}\right)$ which commute with $\iota\left(O_{L}\right)$.

Let $R$ be a hereditary $O_{F}$-order of $D$ with invariant $\vec{f}_{R}$. This means that there exists an isomorphism from $D$ to $\operatorname{Mat}_{m}(\Delta)$ that sends $R$ onto the order $\operatorname{Mat}_{m}\left(\vec{f}_{R}, O_{\Delta}\right)$. Given $g \in D^{\times}$, suppose $g M_{*}\left(\vec{f}_{R}\right)$ is an $\left(O_{L}, O_{\Delta}\right)$-flag in $V$ of type $\left(\iota, \overrightarrow{f_{R}}\right)$. Then we must have

$$
g^{-1} \iota\left(O_{L}\right) g \subset \operatorname{End}_{O_{\Delta}}\left(M_{*}\left(\vec{f}_{R}\right)\right)=R
$$

which means that $g$ is in $\mathcal{E}_{\iota}(R)$ (see (7.1)). Two $\left(O_{L}, O_{\Delta}\right)$-flags $g_{1} M_{*}\left(\overrightarrow{f_{R}}\right)$ and $g_{2} M_{*}\left(\vec{f}_{R}\right)$ in $V$ of type $\left(\iota, \vec{f}_{R}\right)$ are isomorphic if and only if there exists an element $h$ in $C_{\iota}(L)^{\times}$such that $g_{2}^{-1} h g_{1} \in R^{\times}$. If we denote by $\mathbf{F L}\left(O_{L}, O_{\Delta}, m, \iota, \vec{f}\right)$ the set of isomorphism classes of $\left(O_{L}, O_{\Delta}\right)$-flags of a given type $(\iota, \vec{f})$ in $V$, then we conclude that

Proposition 7.5. We have the following bijection

$$
\begin{array}{ccc}
C_{\iota}(L)^{\times} \backslash \mathcal{E}_{\iota}(R) / R^{\times} & \longleftrightarrow & \mathbf{F L}\left(O_{L}, O_{\Delta}, m, \iota, \vec{f}_{R}\right) \\
C_{\iota}(L)^{\times} g R^{\times} & \longmapsto & {\left[g M_{*}\left(\vec{f}_{R}\right)\right] .}
\end{array}
$$

Moreover, let $g M_{*}\left(\vec{f}_{R}\right)$ be an $\left(O_{L}, O_{\Delta}\right)$-flag in $V$ of type $\left(\iota, \vec{f}_{R}\right)$. One has

$$
C_{\iota}(L) \cap g R g^{-1} \cong \operatorname{End}_{\left(O_{L}, O_{\Delta}\right)}\left(g M_{*}\left(\vec{f}_{R}\right)\right)
$$

and

$$
C_{\iota}(L)^{\times} \cap g R^{\times} g^{-1} \cong \operatorname{Aut}_{\left(O_{L}, O_{\Delta}\right)}\left(g M_{*}\left(\vec{f}_{R}\right)\right)
$$

Remark. For any two embeddings $\iota_{1}$ and $\iota_{2}$ of $L$ into $D$, the sets $\mathbf{F L}\left(O_{L}, O_{\Delta}, m, \iota_{1}, \vec{f}_{R}\right)$ and $\mathbf{F L}\left(O_{L}, O_{\Delta}, m, \iota_{2}, \vec{f}_{R}\right)$ are isomorphic if $\iota_{1}$ and $\iota_{2}$ have the same type. 
7.3. Decomposition of $\mathbf{F L}\left(O_{L}, O_{\Delta}, m, \iota, \vec{f}\right)$. In this subsection, we fix an $F$ algebra embedding $\iota$ of $L=L_{1} \times \cdots \times L_{\ell}$ into $D$ of type $\left(m_{1}, \ldots, m_{\ell}\right)$. The centralizer $C_{\iota}(L)$ of $\iota(L)$ in $D$ is canonically isomorphic to

$$
\prod_{w=1}^{\ell} \operatorname{End}_{\left(L_{w}, \Delta\right)}\left(\iota\left(e_{w}\right) V\right)
$$

where $e_{w}$ is the idempotent in $L$ corresponding to $L_{w}$, and $\operatorname{End}_{\left(L_{w}, \Delta\right)}\left(\iota\left(e_{w}\right) V\right)$ is the endomorphism ring of the $\left(L_{w}, \Delta\right)$-bimodule $\iota\left(e_{w}\right) V$, which is a central simple algebra over $L_{w}$.

Let $N_{*}=\left(N_{1}, \ldots, N_{r}\right)$ be an $\left(O_{L}, O_{\Delta}\right)$-flag in $V$ of type $(\iota, \vec{f})$. For each $w$ with $1 \leq w \leq \ell$, we have the chain of lattices

$$
\iota\left(e_{w}\right) N_{1} \supseteq \cdots \iota\left(e_{w}\right) N_{r} \supseteq \iota\left(e_{w}\right) N_{r+1}=\iota\left(e_{w}\right) N_{1} \mathfrak{P}_{\Delta} \text { in } V .
$$

Put $f_{w, i}:=\operatorname{dim}_{\mathbb{F}_{\Delta}}\left(\iota\left(e_{w}\right) N_{i} / \iota\left(e_{w}\right) N_{i+1}\right)$. From $N_{i}=\oplus_{w} \iota\left(e_{w}\right) N_{i}$ it follows that

$$
\sum_{w=1}^{\ell} f_{w, i}=f_{i} \quad \text { and } \quad \sum_{i=1}^{r} f_{w, i}=m_{w} .
$$

We denote by $\vec{f}_{w}^{o}$ the vector obtained by removing zero entries of $\vec{f}_{w}=\left(f_{w, 1}, \ldots, f_{w, r}\right)$, i.e.

$$
\overrightarrow{f_{w}^{o}}=\left(f_{w, j_{1}}, \ldots, f_{w, j_{r_{w}}}\right)
$$

where $1 \leq j_{1}<\cdots<j_{r_{w}} \leq r, f_{w, j_{k}}>0$, and $f_{w, j}=0$ for $j \neq j_{1}, \ldots, j_{r_{w}}$. Then

$$
\iota\left(e_{w}\right) N_{*}:=\left(\iota\left(e_{w}\right) N_{j_{1}}, \ldots, \iota\left(e_{w}\right) N_{j_{r_{w}}}\right)
$$

is an $\left(O_{L_{w}}, O_{\Delta}\right)$-flag in $\iota\left(e_{w}\right) V$ of type $\left(\iota_{w}, \vec{f}_{w}^{o}\right)$, where $\iota_{w}$ is the restriction of $\iota$ on $O_{L_{w}}$.

Conversely, suppose we have an $\left(O_{L_{w}}, O_{\Delta}\right)$-flag $N_{i, *}=\left(N_{w, 1}, \ldots, N_{w, r_{w}}\right)$ in $\iota\left(e_{w}\right) V$ of type $\left(\iota, \overrightarrow{f_{w}^{o}}\right)$ for $1 \leq w \leq \ell$, where $\vec{f}_{w}^{o}$ is obtained by removing zero entries of a vector $\vec{f}_{w}=\left(f_{w, 1}, \ldots, f_{w, r}\right)$, with

$$
\sum_{w=1}^{\ell} f_{w, i}=f_{i} \quad \text { and } \quad \sum_{i=1}^{r} f_{w, i}=m_{w}
$$

Let

$$
N_{i}=\oplus_{w=1}^{\ell} N_{w, i} \subset V, \quad 1 \leq i \leq r .
$$

Then $N_{*}=\left(N_{1}, \ldots, N_{r}\right)$ is an $\left(O_{L}, O_{\Delta}\right)$-flag in $V$ of type $(\iota, \vec{f})$.

It is clear that two $\left(O_{L}, O_{\Delta}\right)$-flags $N_{*}$ and $N_{*}^{\prime}$ in $V$ are isomorphic if and only if $\iota\left(e_{w}\right) N_{*}$ and $\iota\left(e_{w}\right) N_{*}^{\prime}$ are isomorphic $\left(O_{L_{w}}, O_{\Delta}\right)$-flags in $\iota\left(e_{w}\right) V$ for all $w=1, \ldots, \ell$. We get the following result.

Proposition 7.6. (1) One has

$$
\mathbf{F L}\left(O_{L}, O_{\Delta}, m, \iota, \vec{f}\right)=\coprod_{\left(\overrightarrow{f_{1}}, \ldots, \vec{f}_{\ell}\right)}\left(\prod_{w=1}^{\ell} \mathbf{F L}\left(O_{L_{w}}, O_{\Delta}, m_{w}, \iota_{w}, \vec{f}_{w}^{o}\right)\right),
$$

where $\left(\vec{f}_{1}, \ldots, \vec{f}_{\ell}\right)$ runs through all tuples of vectors $\vec{f}_{w}=\left(f_{w, i}\right)_{i} \in \mathbb{Z}_{\geq 0}^{r}$ of nonnegative integers satisfying the condition (7.3), and $\vec{f}_{w}^{o}$ is the vector obtained by removing zero entries of the vector $\vec{f}_{w}$. 
(2) Given an $\left(O_{L}, O_{\Delta}\right)$-flag $N_{*}$ in $V$ of type $(\iota, \vec{f})$, we have

$$
\operatorname{End}_{\left(O_{L}, O_{\Delta}\right)}\left(N_{*}\right)=\prod_{w=1}^{\ell} \operatorname{End}_{\left(O_{L_{w}}, O_{\Delta}\right)}\left(\iota\left(e_{w}\right) N_{*}\right) .
$$

and

$$
\operatorname{Aut}_{\left(O_{L}, O_{\Delta}\right)}\left(N_{*}\right)=\prod_{w=1}^{\ell} \operatorname{Aut}_{\left(O_{L_{w}}, O_{\Delta}\right)}\left(\iota\left(e_{w}\right) N_{*}\right) .
$$

Remark. The above proposition tells us that to understand $\mathbf{F L}\left(O_{L}, O_{\Delta}, m, \iota, \vec{f}\right)$, it suffices to focus on the case when $L$ is a field.

7.4. Special case: when $L$ is an unramified field extension. Fix an embedding $\iota: L \hookrightarrow D$ as usual. In this subsection, we describe explicitly the isomorphism classes in $\mathbf{F L}\left(O_{L}, O_{\Delta}, m, \iota, \vec{f}\right)$ for the case where $L$ is an unramified field extension over $F$. Recall the following basic result:

Proposition 7.7. Let $L$ be a finite extension over $F$. If we let

$$
t:=\operatorname{gcd}([L: F], d),
$$

where $d^{2}=[\Delta: F]$, then the central simple algebra $\Delta \otimes_{F} L$ over $L$ is isomorphic to $\operatorname{Mat}_{t}\left(\Delta^{\prime}\right)$ where $\Delta^{\prime}$ is a central division algebra over $L$ with $\operatorname{dim}_{L} \Delta^{\prime}=(d / t)^{2}$.

From now on, $L$ is an unramified field extension of $K$. Choose an unramified maximal subfield $W$ in $\Delta$. Then $[W: F]=d$. Let $\pi$ be a uniformizer of $F$. There exists an element $u$ in $\Delta$ and a positive integer $\kappa$ with $\operatorname{gcd}(\kappa, d)=1$ such that

$$
u^{d}=\pi^{\kappa} \text { and } u \alpha=\operatorname{Frob}_{W / F}(\alpha) u, \forall \alpha \in W .
$$

Here Frob $W / F$ is the Frobenius automorphism of $W$ over $F$. Therefore $\Delta$ is isomorphic to the cyclic algebra $\left(W / F, \operatorname{Frob}_{W / F}, \pi^{\kappa}\right)$ (cf. [11 $\S 30$ and $\left.\S 31\right)$. The integer $\kappa \bmod d$ is independent of the choices of $W$ and $u$, and

$$
\frac{\kappa}{d} \bmod \mathbb{Z} \in \mathbb{Q} / \mathbb{Z}
$$

is called the Hasse invariant of $D=\operatorname{Mat}_{m}(\Delta)$.

It is known that $\Delta^{\prime}$ is isomorphic to the cyclic algebra

$$
\left(W L / L, \operatorname{Frob}_{W L / L}, \pi^{\kappa^{\prime}}\right)
$$

where $\kappa^{\prime} \equiv \kappa \cdot([L: F] / t) \bmod (d / t)($ cf. [11] (31.9)).

Let $\gamma\left(\right.$ resp. $\left.\gamma^{\prime}\right)$ be a positive integer such that

$$
\gamma \cdot \kappa \equiv 1 \bmod d, \quad\left(\operatorname{resp} \cdot \gamma^{\prime} \cdot \kappa^{\prime} \equiv 1 \bmod (d / t)\right) .
$$

There exists an element $\Pi$ (resp. $\left.\Pi^{\prime}\right)$ in $\Delta$ (resp. $\Delta^{\prime}$ ) such that $\Pi^{d}=\pi$ (resp. $\left.\left(\Pi^{\prime}\right)^{d / t}=\pi\right)$ and

$$
\begin{gathered}
\Pi \alpha=\operatorname{Frob}_{W / F}^{\gamma}(\alpha) \Pi, \quad \forall \alpha \in W \\
\text { (resp. } \left.\Pi^{\prime} \alpha^{\prime}=\operatorname{Frob}_{W L / L}^{\gamma^{\prime}}\left(\alpha^{\prime}\right) \Pi^{\prime}, \quad \forall \alpha^{\prime} \in W L\right) .
\end{gathered}
$$

Obviously,

$$
\Pi^{\prime} \alpha=\operatorname{Frob}_{W / F}^{\gamma \cdot t}(\alpha) \Pi^{\prime} \quad \forall \alpha \in W .
$$


Now, the isomorphism between $\Delta \otimes_{F} L$ and $\operatorname{Mat}_{t}\left(\Delta^{\prime}\right)$ can be described by the following:

$$
\begin{aligned}
& \Pi \otimes 1 \longmapsto\left(\begin{array}{cccc}
0 & 1 & & \\
& \ddots & \ddots & \\
& & 0 & 1 \\
\Pi^{\prime} & & & 0
\end{array}\right) \\
& \alpha \otimes 1 \longmapsto\left(\begin{array}{cccc}
\alpha & & & \\
& \operatorname{Frob}_{W / F}^{\gamma}(\alpha) & & \\
& & \ddots & \\
& & & \operatorname{Frob}_{W / F}^{\gamma(t-1)}(\alpha)
\end{array}\right) \forall \alpha \in W \\
& 1 \otimes \beta \longmapsto\left(\begin{array}{ccc}
\beta & & \\
& \ddots & \\
& & \beta
\end{array}\right) \quad \forall \beta \in L .
\end{aligned}
$$

In particular, denote by $O_{\Delta^{\prime}}$ the maximal compact subring in $\Delta^{\prime}$ and $\mathfrak{P}_{\Delta^{\prime}}:=$ $\Pi^{\prime} O_{\Delta^{\prime}}$, the maximal two-sided ideal in $O_{\Delta^{\prime}}$. Then

$$
O_{\Delta} \otimes_{O_{F}} O_{L} \cong\left(\begin{array}{cccc}
O_{\Delta^{\prime}} & O_{\Delta^{\prime}} & \cdots & O_{\Delta^{\prime}} \\
\mathfrak{P}_{\Delta^{\prime}} & O_{\Delta^{\prime}} & \cdots & O_{\Delta^{\prime}} \\
\vdots & \ddots & \ddots & \vdots \\
\mathfrak{P}_{\Delta^{\prime}} & \cdots & \mathfrak{P}_{\Delta}^{\prime} & O_{\Delta^{\prime}}
\end{array}\right) \subset \operatorname{Mat}_{t}\left(\Delta^{\prime}\right) .
$$

We conclude that

Lemma 7.8. Suppose $L$ is unramified over $F$. Then $O_{\Delta} \otimes_{O_{F}} O_{L}$ is isomorphic to an Iwahori order in $\operatorname{Mat}_{t}\left(\Delta^{\prime}\right)$.

Recall that $\iota: L \hookrightarrow D=\operatorname{End}_{\Delta}(V)$ is a fixed embedding. Then $V$ can be viewed as an $(L, \Delta)$-bimodule via $\iota$. It is natural to think $V$ as a right $\Delta \otimes_{F} L$-module. For $1 \leq i, j \leq t$, let $E_{i, j}$ be the $(i, j)$-th elementary matrix in $\operatorname{Mat}_{t}\left(\Delta^{\prime}\right) \cong \Delta \otimes_{F} L$ (i.e. whose $(i, j)$-entry is one and other entries are zero). Let

$$
V^{(i)}:=V E_{i, i} \quad \text { for } 1 \leq i \leq t .
$$

The multiplication by $E_{i, j}$ from the right gives rise to an isomorphism of free right $\Delta^{\prime}$-modules

$$
\begin{aligned}
\varphi_{j, i}: \quad V^{(i)} & \longrightarrow V^{(j)} \\
x E_{i, i} & \longmapsto x E_{i, j}=\left(\left(x E_{i, i}\right) \cdot E_{i, j}\right) \cdot E_{j, j} .
\end{aligned}
$$

Therefore

$$
m^{\prime}:=\operatorname{rank}_{\Delta^{\prime}} V^{(i)}=\frac{1}{t} \cdot \operatorname{rank}_{\Delta^{\prime}} V=\frac{m[\Delta: F]}{t\left[\Delta^{\prime}: F\right]}=\frac{m \cdot t}{[L: F]}
$$

as $t^{2}\left[\Delta^{\prime}: F\right]=\left[\Delta \otimes_{F} L: F\right]=[\Delta: F][L: F]$.

Lemma 7.9. For $1 \leq i \leq t$, we have

$$
\begin{array}{ccc}
\operatorname{End}_{\Delta \otimes_{F} L}(V) & \cong & \operatorname{End}_{\Delta^{\prime}}\left(V^{(i)}\right) \\
g & \left.\mapsto g\right|_{V^{(i)}} .
\end{array}
$$


Proof. For a given $h \in \operatorname{End}_{\Delta^{\prime}}\left(V^{(i)}\right)$, consider the following map

$$
\Phi_{h}:=\sum_{j=1}^{t} \varphi_{j, i} \circ h \circ \varphi_{i, j}: \bigoplus_{j=1}^{t} V^{(j)} \longrightarrow \bigoplus_{j=1}^{t} V^{(j)} .
$$

It is clear that $\Phi_{h} \in \operatorname{End}_{\Delta \otimes_{F} L}(V)$ and $\left.\Phi_{h}\right|_{V^{(i)}}=h$.

On the other hand, let $g \in \operatorname{End}_{\Delta_{\otimes_{F}} L}(V)$. For any $\alpha$ in $V$,

$$
g(\alpha)=g\left(\sum_{j=1}^{t} \alpha E_{j, j}\right)=\sum_{j=1}^{t}\left(g\left(\alpha E_{j, i}\right)\right) E_{i, j}=\sum_{j=1}^{t}\left(\varphi_{j, i} \circ\left(\left.g\right|_{V^{(i)}}\right) \circ \varphi_{i, j}\right)(\alpha)
$$

and the result follows.

For $1 \leq i<t$, one has

$$
\Pi^{i} \cdot E_{t, t}=E_{t-i, t} \text { and } \Pi^{t} \cdot E_{t, t}=E_{t, t} \cdot \Pi^{\prime} .
$$

Let $N_{*}=\left(N_{1}, \ldots, N_{r}\right)$ be an $\left(O_{L}, O_{\Delta}\right)$-flag in $V$ of type $\vec{f}=\left(f_{1}, \ldots, f_{r}\right)$. Then we can view $N_{*}$ as a flag of right $O_{\Delta} \otimes_{O_{F}} O_{L}$-modules (in $V$ ). Set

$$
N_{i, j}:=N_{i} \cdot E_{t+1-j, t} \subset V^{(t)} \text { for } 1 \leq i \leq r \text { and } 1 \leq j \leq t .
$$

Then multiplying $E_{t, t}$ (from the right) on the chain

$$
N_{1} \supsetneq N_{2} \supsetneq \cdots \supsetneq N_{r} \supsetneq N_{r+1}=N_{1} \cdot \Pi,
$$

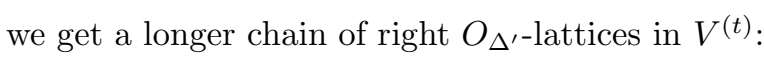

$$
\begin{aligned}
& \begin{array}{lllllll}
N_{1,1} & \supseteq N_{2,1} & \supseteq & \cdots & \supseteq & N_{r, 1}
\end{array} \\
& \supseteq N_{1,2} \supseteq N_{1,2} \supseteq N_{2,} \quad \cdots \quad \supseteq N_{r, 2} \\
& \begin{array}{llllll} 
& \cdots & \cdots & \cdots & \ldots
\end{array} \\
& \supseteq N_{1, t} \quad \supseteq N_{2, t} \supseteq N_{1,} \quad \supseteq N_{r, t}
\end{aligned}
$$

Moreover,

$$
N_{i}=\bigoplus_{j=1}^{t} N_{i, j} E_{t, t+1-j} \quad \text { for } 1 \leq i \leq r .
$$

Recall that $\mathbb{F}_{\Delta}=O_{\Delta} / \mathfrak{P}_{\Delta}$ and we let $\mathbb{F}_{\Delta^{\prime}}=O_{\Delta^{\prime}} / \mathfrak{P}_{\Delta^{\prime}}$. Let

$$
f_{i, j}:= \begin{cases}\operatorname{dim}_{\mathbb{F}_{\Delta^{\prime}}}\left(N_{i, j} / N_{i+1, j}\right) & \text { for } 1 \leq i<r, \\ \operatorname{dim}_{\mathbb{F}_{\Delta^{\prime}}}\left(N_{r, j} / N_{1, j+1}\right) & \text { for } i=r \text { and } 1 \leq j<t, \\ \operatorname{dim}_{\mathbb{F}_{\Delta^{\prime}}}\left(N_{r, t} /\left(N_{1,1} \Pi^{\prime}\right)\right) & \text { for } i=r \text { and } j=t .\end{cases}
$$

Then for $1 \leq i \leq r$,

$$
\left[\mathbb{F}_{\Delta^{\prime}}: \mathbb{F}_{\Delta}\right] \cdot \sum_{j=1}^{t} f_{i, j}=\frac{[L: F]}{t} \cdot \sum_{j=1}^{t} f_{i, j}=f_{i} .
$$

This tells us that $[L: F] / t$ divides $f_{i, j}$ for all $i$.

We fix an order of the index set $\{(i, j)\}_{1 \leq i \leq r, 1 \leq j \leq t}$ :

$$
(i, j)<\left(i^{\prime}, j^{\prime}\right) \text { if }\left\{\begin{array}{l}
j<j^{\prime} \\
j=j^{\prime}
\end{array} \text { and } i<i^{\prime} .\right.
$$

Consider the vector

$$
\vec{f}_{*}=\left(f_{i, j}\right)_{1 \leq i \leq r, 1 \leq j \leq t}=\left(f_{1,1}, \ldots, f_{r, 1}, f_{1,2}, \ldots, f_{r, 2}, \ldots, f_{1, t}, \ldots, f_{r, t}\right) .
$$


Let $\vec{f}_{*}^{o}$ be the vector obtained by removing the zero entries of the vector $\vec{f}_{*}$. Picking the "gap" of the chain $\left(N_{i, j}\right)_{1 \leq i \leq r, 1 \leq j \leq t}$ as (7.4), we obtain an $O_{\Delta^{\prime}}$-flag in $V^{(t)}$ of type $\vec{f}_{*}^{o}$. It is clear that

$$
\operatorname{End}_{E\left(O_{L}, O_{\Delta}\right)}\left(N_{*}\right) \cong \operatorname{Mat}_{m^{\prime}}\left(\vec{f}_{*}^{o}, O_{\Delta^{\prime}}\right) \subset \operatorname{Mat}_{m^{\prime}}\left(\Delta^{\prime}\right) .
$$

Conversely, suppose $[L: F] / t$ divides $f_{i}$ for all $i$. Then for any such a chain of $O_{\Delta^{\prime}}$-lattices $\left(N_{i, j}\right)_{1 \leq i \leq r, 1 \leq j \leq t}$ in $V^{(t)}$, we can rebuild an $\left(O_{L}, O_{\Delta}\right)$-flag $N_{*}=$ $\left(N_{1}, \ldots, N_{r}\right)$ in $V$ of type $(\iota, \vec{f})$ by setting

$$
N_{i}=\bigoplus_{j=1}^{t} N_{i, j} \cdot E_{t, t+1-j} \subset \bigoplus_{j=1}^{t} V^{(t+1-j)}=V .
$$

Two $\left(O_{L}, O_{\Delta}\right)$-flags $N_{*}$ and $N_{*}^{\prime}$ are isomorphic if and only if $f_{i, j}=f_{i, j}^{\prime}$ for $1 \leq i \leq r$ and $1 \leq j \leq t$. We conclude the above discussion in the following result:

Theorem 7.10. Suppose an embedding $\iota: L \hookrightarrow D$ is given, where $L$ is an unramified extension over $F$.

(1) The set $\mathbf{F L}\left(O_{L}, O_{\Delta}, m, \iota, \vec{f}\right)$ of isomorphism classes of $\left(O_{L}, O_{\Delta}\right)$-flags in $V$ of type $\left(\iota, \vec{f}=\left(f_{1}, \ldots, f_{r}\right)\right)$ can be parametrized by the vectors $\vec{f}_{*}=\left(f_{i, j}\right)_{1 \leq i \leq r, 1 \leq j \leq t}$ of non-negative integers $f_{i, j}$ satisfying

$$
\frac{[L: F]}{t} \cdot \sum_{j=1}^{t} f_{i, j}=f_{i} \quad \text { for all } 1 \leq i \leq r .
$$

(2) Let $N_{*}$ be an $\left(O_{L}, O_{\Delta}\right)$-flag in $V$ of type $\vec{f}$. Let $\vec{f}_{*}=\left(f_{i, j}\right)_{1 \leq i \leq r, 1 \leq j \leq t}$ be its corresponding vector. Then

$$
\operatorname{End}_{\left(O_{L}, O_{\Delta}\right)}\left(N_{*}\right) \cong \operatorname{Mat}_{m^{\prime}}\left(\vec{f}_{*}^{o}, O_{\Delta^{\prime}}\right) \subset \operatorname{Mat}_{m^{\prime}}\left(\Delta^{\prime}\right)
$$

where the vector $\vec{f}_{*}^{o}$ is obtained by removing zero entries of $\vec{f}_{*}$.

Acknowledgments. The authors would like to thank E.-U. Gekeler and Jing Yu for their steady interest and encouragements. The paper was revised while the second named author visited the IEM, Universität Duisburg-Essen. He wishes to thank the institution for kind hospitality and good working conditions. The first named author was partially supported by the grant NSC 100-2811-M-007-046. The second named author was partially supported by the grants NSC 100-2628-M-001006-MY4 and AS-99-CDA-M01.

\section{REFERENCES}

[1] A. Brumer, Structures of hereditary orders, Bull. Amer. Math. Soc. 69 (1963), 721-724. addendum, ibid. 70 (1964), 185.

[2] J. Brzezinski, On automorphisms of quaternion orders. J. Reine Angew. Math. 403 (1990), $166-186$

[3] V. Drinfeld, Elliptic modules, (English translation) Math. USSR-Sb. 23 (1976), 561-592.

[4] M. Denert and J. Van Geel, The class number of hereditary orders in non-Eichler algebras over global function fields. Math. Ann. 282 (1988), no. 3, 379-393.

[5] M. Eichler, Zur Zahlentheorie der Quaternionen-Algebren. J. Reine Angew. Math. 195 (1955), 127-151.

[6] E.-U. Gekeler, On finite Drinfeld modules. J. Algebra 141 (1991), 187-203. 
[7] E.-U. Gekeler, On the arithmetic of some division algebras. Comment. Math. Helv. 67 (1992), 316-333.

[8] M. Papikian, Endomorphism of exceptional D-elliptic sheaves, Math. Z. 266 (2010), 407-423.

[9] G. Prasad, Volumes of $S$-arithmetic quotients of semi-simple groups. Inst. Hautes Études Sci. Publ. Math. 69 (1989), 91-117.

[10] A. Pizer, On the arithmetic of quaternion algebras. Acta Arith. 31 (1976), no. 1, 61-89.

[11] I. Reiner, Maximal orders. London Mathematical Society Monographs, No. 5. Academic Press, London-New York, 1975. 395 pp.

[12] S.-C. Shih, T.-C. Yang and C.-F. Yu, Embeddings of fields in simple algebras over global fields. arXiv:1108.0830 $27 \mathrm{pp}$.

[13] M.-F. Vignéras, Arithmétique des algèbres de quaternions. Lecture Notes in Math., vol. 800, Springer-Verlag, 1980.

[14] F.-T. Wei and C.-F. Yu, Mass formula of division algebras over global function fields. J. Number Theory 132 (2012), 1170-1184.

[15] C.-F. Yu, Embeddings of fields into simple algebras: generalizations and applications. J. Algebra 368 (2012), 1-20.

[16] C.-F. Yu and J. Yu, Mass formula for supersingular Drinfeld modules. C. R. Acad. Sci. Paris Sér. I Math. 338 (2004) 905-908.

(Wei) Department of Mathematics, National Tsing-Hua University, Hsinchu 30013, TAIWAN

E-mail address: ftwei@mx.nthu.edu.tw

(Yu) Institute of Mathematics, Academia Sinica and NCTS (Taipei Office), 6th Floor, Astronomy Mathematics Building, No. 1, Roosevelt Rd. Sec. 4, Taipei 10617, Taiwan

E-mail address: chiafu@math.sinica.edu.tw 